\title{
A Bayesian Filtering Approach to Incorporate 2D/3D Time-lapse Confocal Images for Tracking Angiogenic Sprouting Cells Interacting with the Gel Matrix.
}

\author{
Lee-Ling S. Ong ${ }^{\mathrm{a}, *}$, Justin Dauwels ${ }^{\mathrm{b}}$, Marcelo H. Ang Jr ${ }^{\mathrm{c}}$, H. Harry Asada ${ }^{\mathrm{a}, \mathrm{d}}$ \\ ${ }^{a}$ Singapore-MIT Alliance for Research and Technology,Singapore. \\ ${ }^{b}$ Department of Electrical and Electronic Engineering, Nanyang Technological University, Singapore. \\ ${ }^{c}$ Department of Mechanical Engineering, National University of Singapore, Singapore. \\ ${ }^{d}$ Department of Mechanical Engineering, MIT, Cambridge, MA, USA.
}

\begin{abstract}
We present a new approach to incorporating information from heterogeneous images of migrating cells in $3 D$ gel. We study $3 D$ angiogenic sprouting, where cells burrow into the gel matrix, communicate with other cells and create vascular networks. We combine time-lapse fluorescent images of stained cell nuclei and transmitted light images of the background gel to track cell trajectories. The nuclei images are sampled less frequently due to photo toxicity. Hence, $3 D$ cell tracking can be performed more reliably when $2 D$ sprout profiles, extracted from gel matrix images, are effectively incorporated. We employ a Bayesian filtering approach to optimally combine the two heterogeneous images with different sampling rates. We construct stochastic models to predict cell locations and sprout profiles and condition the likelihood of nuclei location by the sprout profile. The conditional distribution is non-Gaussian and the cell dynamics is non-linear. To jointly update cell and sprout estimates, we use a Rao-Blackwell particle filter. Simulation and experimental results show accurate tracking of multiple cells along with sprout formation, demonstrating synergistic effects of incorporating the two types of images.
\end{abstract}

Keywords: Cell tracking, Recursive Bayesian filtering, Rao-Blackwell particle filter, Confocal microscopy, Time-lapse microscopy, Angiogenesis

\section{Introduction}

Cells in the human body migrate to perform numerous biological functions, including development and wound healing. For years, cell migration has been studied with two-dimensional $(2 D)$ on-the-gel assays using $2 D$ imaging and cell tracking techniques (Abercrombie et al., 1970). When cells migrate in three-dimensional $(3 D)$ environments, they exhibit strikingly different behaviors (Cukierman et al., 2001; Petrie and Yamada, 2012). In $3 D$, cells migrate by remodeling and cleaving the surrounding gel whereas in $2 D$, they simply move across a free surface (Yamada and Cukierman, 2007). However, their migration behavior and interactions with the surrounding gel are still poorly understood. To help understand $3 D$ migration and cell-gel interactions, we aim to develop a new automated image processing methodology.

*Tel.: +65 65164161; E-mail address: sharon.ong@ smart.mit.edu (S. Ong).

Preprint submitted to Medical Image Analysis
We develop our methodology specifically for angiogenesis, the growth process of blood vessels from existing blood vessels. These vessels are lined with endothelial cells. In $3 D$ angiogenesis, an endothelial tip cell extends its filopodia into the gel matrix in response to local chemo-attractants and mechanical stimuli. The cell then moves in the direction of the filopodia and creates a lumen in the gel (Folkman and Haudenschild, 1980). Other endothelial cells, called stalk cells follow the tip cell and migrate within the lumen. Their coordinated migration can lead to successful formations of blood vessel networks.

The angiogenic environment is mimicked by in vitro $3 D$ microfluidic assays (Vickerman et al., 2008), which allow more detailed experiments and observations. We expect that valuable experimental studies will be performed with these assays to answer important biological questions such as; how sprouting cells burrow into $3 D$ gel, how they interact with each other and with the surrounding gel, and how they migrate in a coordinated manner to form a sprout (Gerhardt, 2006). Such detailed 


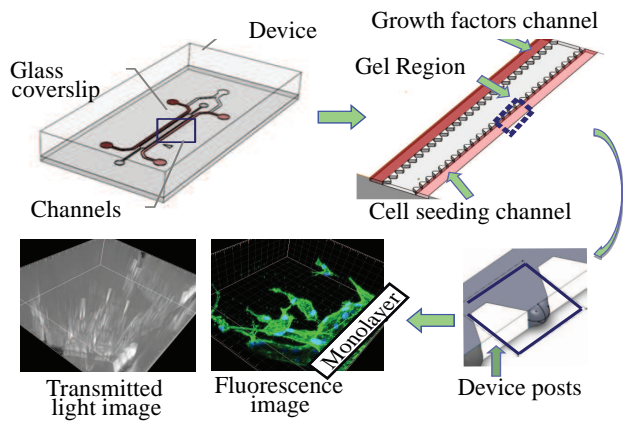

Figure 1: Experiments are carried out in a microfluidic flow chamber with 37 migration regions. We inject collagen gel between the two channels. Cells are seeded in one channel and growth factors are introduced in the other. A confocal microscope captures fluorescence and transmitted light images of each migration region, including the cell monolayer. Image adapted from (Farahat et al., 2012)).

studies entail advanced image analysis to track and characterize individual cells, gel remodeling and the formation of vascular sprouts.

This paper presents an approach to simultaneous cell and vascular sprout tracking; fusing nuclei images with gel matrix images. Our methodology can optimally (a) combine data from heterogeneous images and known biological models, (b) track cell movements despite a low sampling rate of $3 D$ nuclei position and (c) detect changes in vascular geometry. Although we have developed our methodology specifically for angiogenic sprouting, our framework can be generalized and applied to other applications which involve interactions between migrating cells and the surrounding gel. These applications include stem cell differentiation and myogenesis. Stem cells differentiate into many cells including myoblasts, which collectively form tissues. In myogenesis, myoblasts migrate and fuse into myotubes. We can acquire the $2 D$ shape profile of the myotubes from transmitted light images and $3 D$ nuclei location from confocal fluorescence images (Sakar et al., 2012). By applying our joint cell-sprout tracking system, we can simultaneously track each nuclei and the overall shape of the tubes/tissue formation.

\subsection{Cell-Matrix Interactions and 2D/3D Images}

In our microfluidic device (Farahat et al., 2012), shown in Figure 1, we inject collagen gel between the two channels. Through one channel,we seed cells, which attach to one side of the collagen and form a monolayer. Prior to seeding, the cell nuclei were marked with fluorescent dye. Angiogenic growth factors, supplied in the other channel, diffuse through the collagen gel. These factors drive the cells to prolifer- ate and migrate into the gel. Figure 2(a) illustrates images acquired from an experiment of endothelial cells sprouting in a micro-fluidic assay. Nuclei locations of the migrating cells are extracted from $3 D$ confocal fluorescent images ( $a 1$ and $a 2$ ). The corresponding brightfield transmitted light image (a3) shows the sprout profile created in the gel matrix (a4). Both images are captured by the same microscope at the same time and are registered by default. By correlating these two images (a5), we estimate cell nuclei locations and sprout shape more reliably than doing so separately. In Figure 2(b), we visualize the correlated sprout shape and cell positions. We classify the cells to tip and stalk cells and the sprout to lumens and filopodia.

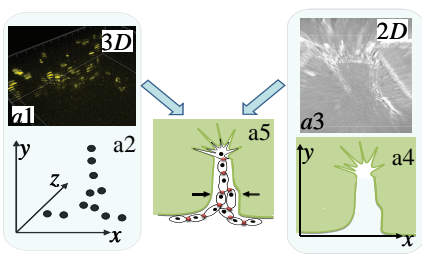

(a) Fusing Time Lapse Images

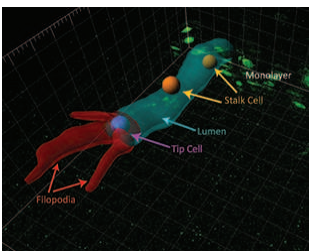

(b) Visualization of a sprout group
Figure 2: Incorporating $3 D$ with $2 D$ information. (a) Adapted from (Ong et al., 2011b), 3D cell nuclei centroid locations ( $a 2)$ are obtained from the fluorescent images $(a 1)$. The bright field images $(a 3)$ provide $2 D$ information about the sprout (a4). The goal is to combine this information (a5). In the sprout group visualized in (b), we visualize a lumen (cyan tube), created by a tip cell (purple sphere). Tip cells are characterized by extensive filopodia, indicated in red. They are followed by stalk cells (orange spheres), which migrate within the lumen.

The $3 D$ location information of migrating cells is obtained from confocal fluorescent images. Acquiring these images at a high sampling rate is harmful to cells. Images are acquired by scanning many thin sections of the cell at different focal planes. These multiple exposures accelerate photochemical destruction in the cell. As a result, $3 D$ confocal images are available only at a low sampling rate. This may compromise reliable tracking of cell movements. Migrating cells often exhibit complex behaviors. Some cells move faster and pass other cells while some cells proliferate. During angiogenic sprouting, some cells create new sprout branches, moving away from others. When nuclei locations are sampled at a low rate, we fail to track these cell movements accurately.

On the other hand, we can observe the sprout conformation in the gel from transmitted light images, which can be acquired at a high sampling rate. Each transmitted light image is subjected to out-of-focus blur. Fur- 
thermore, the $z$-axis resolution of $7 \mu \mathrm{m}$ between each focal plane is poor, when compared to the $x y$-axes resolution of $0.684 \mu \mathrm{ms}$ per pixel. Hence, a sprout's $z-$ coordinates are not obtained and its observation is considered $2 D$. Nevertheless, this data is useful when updated to $3 D$ cell trajectory estimation. With this data, we can also detect false nuclei candidates segmented from fluorescence images, reducing false track updates. Filopodia and lumens are identified from the sprout profile. Detecting filopodia allows us to determine the cells' phenotype; a tip or stalk cell, which in turn, is used to detect new sprout branching. Furthermore, the micro-fluidic assays are "shallow", with a depth of only $120 \mu \mathrm{m}$ in the $z$ direction, compared to $14800 \mu \mathrm{m}$ and $1300 \mu \mathrm{m}$ in the $x$ and $y$ direction respectively. We observed that lumens extend mostly in the $x y$-plane. However, we track the cells in $3 D$ for better cell-to-track associations. Cells with overlapping $x$ and $y$ coordinates tend to have different $\mathrm{z}$-axis coordinates.

\subsection{Related Work}

Various methods for cell tracking have been studied by many groups (Debeir et al., 2005; Padfield et al., 2009; Bise et al., 2011). Techniques applied include using a "nearest-neighbor association" or mean shift process (Debeir et al., 2005; Bise et al., 2011) and a combination of active contours and optical flow (Montrucchio et al., 2005; Sacan et al., 2008; Padfield et al., 2009). Vessel segmentation algorithms extract parameters including centerlines, edges and junctions and the whole vascular network (Kirbas and Quek, 2000; Lesage et al., 2009). Techniques applied include pattern recognition techniques (Zana and Klein, 2001; Truc et al., 2009), model-based approaches (Agin and Binford, 1976; Pellot et al., 1994) and tracking-based approaches in the spatial frame (Florin et al., 2006; Wörz and Rohr, 2007).

Probabilistic methods optimally perform cell segmentation and association in Bayesian framework and resolve cell association uncertainties in cases of high cell densities and velocities. Particle filtering (a probabilistic method) was used in (Smal et al., 2008; Godinez et al., 2009) for molecular level tracking. At the cellular level, Kalman filtering (Yang et al., 2006), Multiple Hypothesis Tracking (MHT) (Bunyak et al., 2006) and Interacting Multiple Models (IMM) (Li et al., 2008) have been applied for tracking. Majority of these probabilistic approaches are applied on $2 D$ cell migration and only used either fluorescence or transmitted light images.

To our knowledge, our work is the only method combining $3 D$ cell tracking with $2 D$ vessel tracking in the spatial and temporal domain. To combine the time lapse images of cell and gel in an optimal manner, we apply a Bayesian filtering framework presented in Section 2. A joint probability distribution, comprising of cell and sprout profile variables, is propagated through time using dynamic equations based on known biological models. Estimation of cell locations is conditioned by observed sprout profile data, as the cells are likely to be on the wall of a lumen or within the lumen. The conditional distribution is non-Gaussian. We apply a Rao-Blackwell particle filter, which allows simultaneous updates of cell and sprout parameters in an efficient manner. We assess our methods on a synthetic data set in Section 3. Image processing techniques applied are described in Appendix A. Our approach is then demonstrated on experimental results in Section 4. We conclude in Section 5, summarizing the advantages of our methodology.

\section{Methods}

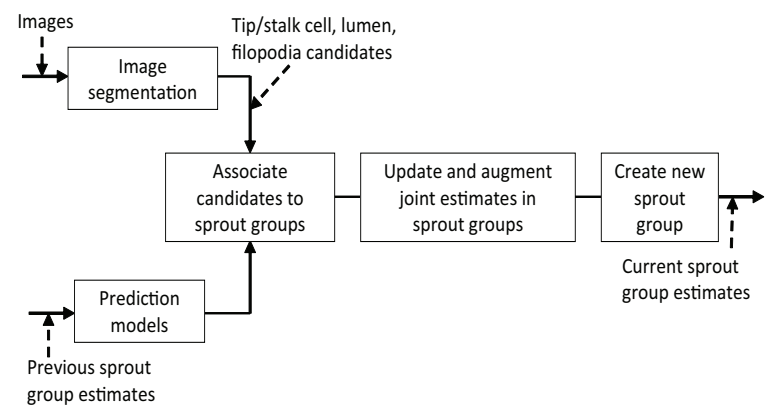

Figure 3: Overview of the tracking system. Multiple sprouts, an example is shown in Figure 2(b), are treated as separate groups of cells, lumen and filopodia. We predict these parameters using motion models. The segmented nuclei, lumen and filopodia candidates are associated firstly with a particular sprout, and then with existing tracks in its joint estimate. Matched candidates are updated to an existing track. New sprouts may be created for dissociated sprout profiles. New nuclei, lumen and filopodia tracks in a sprout may be augmented to a joint estimate. Tracks and sprouts may also be terminated.

Our tracking system is summarized in Figure 3. We use Bayesian filtering to optimally track the complete spatiotemporal history of sprout formation. This includes nuclei centroid positions and velocities, cell phenotypes, as well as the location and shape of lumens and filopodia. The cell proliferation history is also tracked. Multiple sprouts are treated as separate groups of cells and a lumen, jointly estimated from segmented images. Each sprout is tracked individually. 
Tip Cells: Tip cells burrow into the gel to create a lumen. Their behavior is stochastic but a momentum effect is exhibited, as their velocities tend to be maintained (Borau et al., 2011).

Stalk Cells: Stalk cells tend to crawl along the lumen wall. When the lumen is narrow, the cells tend to move in the middle of the lumen. For a wide lumen, they tend to move along one side of the wall (Kim et al., 2012). We model the cell desired heading conditioned on lumen parameters.

Lumen: Tip cells secret protease, such as matrix metalloproteinases (MMPs), that degrades the gel matrix to widen the lumen. We model the amount of protease released and therefore the lumen radius increase, to be proportional to the speed of the tip cell (Wood et al., 2012).

Filopodia: Rapid movements of filopodia are characteristics of a tip cell. The filopodia dynamics are not modeled as the underlying mechanisms are unknown.

Table 1: Summary of models for predicting cell and sprout components.

We compare new observations and existing sprouts estimated previously. From this association, we would (a) update the state of the existing sprout, (b) add a new sprout to the groups to be tracked, or (c) terminate tracking an existing sprout from the groups. In (a), the update of the state is made based on the discrepancy between the new observation and a predicted state of each sprout. Cell nuclei position, as well as the location and shape of each lumen and filopodia, are propagated from a previously estimated state to a new state using stochastic models. The models for predicting cell movements are based on lumen growth and cell dynamics, available in the literature (Kim et al., 2012; Wood et al., 2012).

\subsection{Formulation of Tracking Problem}

Here we consider the problem of jointly estimating the cell state $\mathbf{X}_{k}$ and sprout profile $\mathbf{M}_{k}$, based on nuclei observations $\mathbf{Z}_{1: k}^{3 D}$, sprout profile observations $\mathbf{Z}_{1: k}^{2 D}$, exogenous inputs driving the migrating cells $\mathbf{U}_{1: k}^{\mathbf{X}}$ and inputs driving the sprout evolution $\mathbf{U}_{1: k}^{\mathbf{M}}$, to obtain the probability distribution function (PDF),

$$
p\left(\mathbf{X}_{k}, \mathbf{M}_{k} \mid \mathbf{Z}_{1: k}^{2 D}, \mathbf{Z}_{1: k}^{3 D}, \mathbf{U}_{1: k}^{\mathbf{X}}, \mathbf{U}_{1: k}^{\mathbf{M}}\right) .
$$

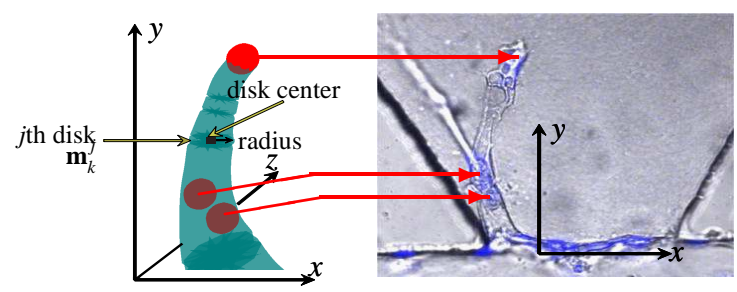

Figure 4: Cell and sprout representation (left) of an acquired image (right). The nuclei (red dots) are represented by their $3 D$ centroid coordinates. The lumen (blue tube) is parameterized by its centerline coordinates and radius.

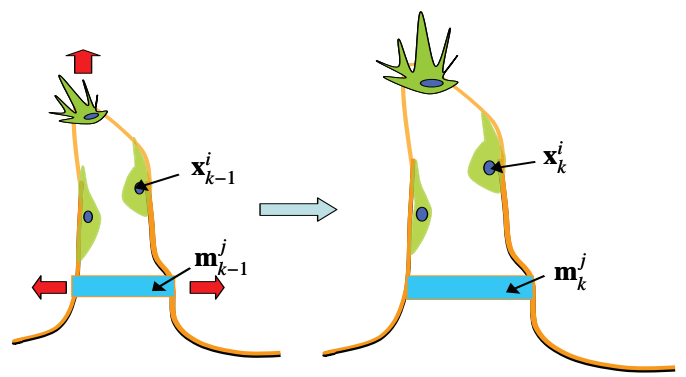

Figure 5: A representation of the lumen profile from time $k-1$ to time $k$ is shown. At time $k$, lumen disks expand from $\mathbf{m}_{k-1}^{j}$ to $\mathbf{m}_{k}^{j}$. At the same time, the cell nuclei at $\mathbf{x}_{k-1}^{i}$ migrates along the lumen to $\mathbf{x}_{k}^{i}$. An updated nuclei position may be inferred from observations of lumen parameters.

We denote $\mathbf{x}_{k}^{i} \in \mathbb{R}^{3}$ is the Cartesian coordinates of the $i$ th cell at time $k$, shown in Figure 4. Each cell is also parameterized by a tip/stalk cell phenotype. For $n$ migrating cells within the image frame, the collective representation is $\mathbf{X}_{k}=\left[\mathbf{x}_{k}^{1}, \ldots, \mathbf{x}_{k}^{n}\right]^{T} \in \mathbb{R}^{3 n}$. The number of migrating cells varies as the cells proliferate or die during the experiment.

We represent the geometry of each filopodia and lumen using medial axis transforms (MAT) (Sherbrooke et al., 1996). We denote $\mathbf{m}_{k}^{j} \in \mathbb{R}^{3}$ to be a parameter vector describing the center coordinates and shape of the $j$ th disk of a filopodia or lumen MAT at time $k$, as shown in Figure 4. All disks associated with a MAT, is given by $\mathbf{M}_{k}=\left[\mathbf{m}_{k}^{1}, \ldots, \mathbf{m}_{k}^{l}\right]^{T} \in \mathbb{R}^{3 n}$. When lumens and filopodia extend, new medial axis disks are added. This increases the vector $\mathbf{M}_{k}$ accordingly. Collectively, we define the state vector as $\mathbf{x}_{k}^{S}=\left[\mathbf{X}_{k}, \mathbf{M}_{k}\right]$.

The lumen dynamics is linear while the cell dynamics are non-linear. The joint lumen and cell states are propagated through dynamic models with an extended Kalman filter. Therefore, we model the joint state $\mathbf{x}_{k}^{\mathbf{S}}$ by 
means of a Gaussian distribution:

$$
p\left(\mathbf{x}_{k}^{\mathbf{S}} \mid \mathbf{Z}_{1: k}^{2 D}, \mathbf{Z}_{1: k}^{3 D}, \mathbf{U}_{k}^{\mathbf{X}}, \mathbf{U}_{k}^{\mathbf{M}}\right)=\mathcal{N}\left(\mathbf{x}_{k}^{\mathbf{S}} ; \hat{\mathbf{x}}_{k \mid k-1}^{\mathbf{S}}, \mathbf{P}_{k \mid k-1}^{\mathbf{x}, \mathbf{S}}\right),
$$

Here, the joint mean vector and covariance matrix are $\hat{\mathbf{x}}_{k \mid k-1}^{\mathbf{S}}$ and $\mathbf{P}_{k \mid k-1}^{\mathbf{x}, \mathbf{S}}$.

Figure 5 shows a representation of cells and lumen disks over a lumen outline. The cell $\mathbf{x}^{i}$ migrates and the lumen disk $\mathbf{m}^{j}$ expands in that time period. As an analogy to robotics, we can associate migrating cells to robotic vehicles and the lumen shape to a map of the field. The collective cell and lumen tracking problem may be formulated as a popular robotic technique known as SLAM (Simultaneous Localization and Mapping) (Leonard and Durrant-Whyte, 1991). In SLAM, the map parameters are updated simultaneously with the robot's parameters, aiding the estimate of the robot's location. However, the problem is fundamentally different in two ways:

- The lumen shape evolves dynamically as a time function. However, the landmarks in the SLAM map are, in general, time invariant.

- The cell activities directly affect the lumen formation, whereas the robotic vehicles do not alter the map.

We formulate a simultaneous cell and lumen profile tracking problem which exploits these bi-directional interactions.

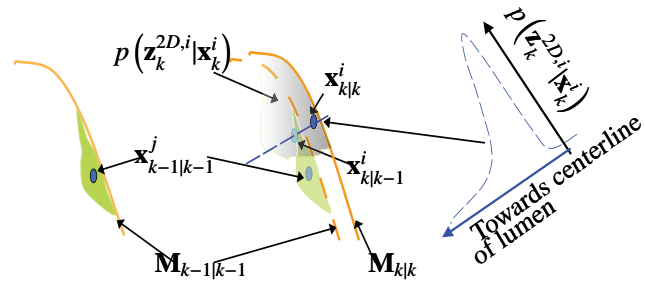

Figure 6: An illustration of the update of cell parameters given lumen observations. First, the nuclei position $\mathbf{x}_{k-1 \mid k-1}^{j}$, is predicted forward in time to $k$ based on the lumen estimate information at time $k-1$. Next, the cell parameters are updated with the new lumen observation, $\mathbf{z}_{k}^{2 D, i}$, to obtain state $\mathbf{x}_{k \mid k}^{i}$. There is a higher probability that the cell is on the lumen wall. Therefore, the likelihood function $p\left(\mathbf{z}_{k}^{2 D, i} \mid \mathbf{x}_{k}^{i}\right)$, is a skewed normal distribution, orthogonal to the lumen slope.

Nuclei locations from the $3 D$ confocal image are represented by $\mathbf{Z}_{k}^{3 D}$ whereas $\mathbf{Z}_{k}^{2 D}$ represents the lumen and filopodia disks extracted from transmitted light images. We illustrate the non-Gaussian relationship between joint lumen and cell parameters from time $k-1$ to $k$ in Figure 6 . The cell parameters are predicted forward in time to $k$ based on the lumen information at time $k-1$. As the lumen diameter increases, the cell parameters would be updated to reflect this increase. There is a higher probability that the cell is on the lumen wall. Therefore, to update nuclei coordinates, the variations in lumen profile are considered.

\subsection{Prediction Dynamics}
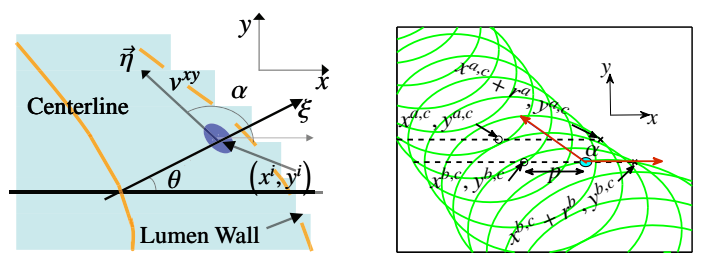

(a) Kinematic arrangement of (b) Derivation of $\alpha$ from lunuclei motion men parameters

Figure 7: Prediction of nuclei motion conditioned on lumen parameters. (a) The cell crawls on the lumen wall at a direction of $\vec{\eta}$, its heading angle steered by the tangent angle of the lumen $\alpha$ at the location of adhesion. (b) shows the parameters of two lumen disks $\mathbf{m}^{a}$ and $\mathbf{m}^{b}$. Here $\mathbf{m}^{a}=\left[x^{a, c}, y^{a, c}, r^{a}\right]$ and $\mathbf{m}^{b}=\left[x^{b, c}, y^{b, c}, r^{b}\right]$ are the disks that minimize the Euclidean distance between the cell $y$-coordinate and that of the MAT disk centre. We then determine the tangent angle $\alpha$ from two points on the lumen wall, $\left[x^{b, c}+r^{b}, y^{b, c}\right]$ and $\left[x^{a, c}+r^{a}, y^{a, c}\right]$.

The PDF (Equation 1) can be propagated based on dynamic models of both nuclei positions and lumen shape:

$$
\begin{aligned}
& p\left(\mathbf{x}_{k}^{\mathbf{S}} \mid \mathbf{Z}_{1: k-1}^{3 D}, \mathbf{Z}_{1: k-1}^{2 D}, \mathbf{U}_{1: k}^{\mathbf{X}}, \mathbf{U}_{1: k}^{\mathbf{M}}\right)= \\
& \int p\left(\mathbf{x}_{k}^{\mathbf{S}} \mid \mathbf{x}_{k-1}^{\mathbf{S}}, \mathbf{U}_{k}^{\mathbf{X}}, \mathbf{U}_{k}^{\mathbf{M}}\right) \\
& p\left(\mathbf{x}_{k-1}^{\mathbf{S}} \mid \mathbf{Z}_{1: k-1}^{3 D}, \mathbf{Z}_{1: k-1}^{2 D}, \mathbf{U}_{1: k-1}^{\mathbf{X}}, \mathbf{U}_{1: k-1}^{\mathbf{M}}\right) d \mathbf{x}_{k-1}^{\mathbf{S}},
\end{aligned}
$$

where $p\left(\mathbf{x}_{k-1}^{\mathbf{S}} \mid \mathbf{Z}_{1: k-1}^{3 D}, \mathbf{Z}_{1: k-1}^{2 D}, \mathbf{U}_{1: k-1}^{\mathbf{X}}, \mathbf{U}_{1: k-1}^{\mathbf{M}}\right)$ is the joint probability density at time $k-1$, and $p\left(\mathbf{x}_{k}^{\mathbf{S}} \mid \mathbf{x}_{k-1}^{\mathbf{S}}, \mathbf{U}_{k}^{\mathbf{X}}, \mathbf{U}_{k}^{\mathbf{M}}\right)$ is the joint state transition model. Using Bayes rule, we decompose the joint probability in Equation 1 to:

$$
p(\mathbf{X}, \mathbf{M}, \cdot)=p(\mathbf{X} \mid \mathbf{M} \cdot) p(\mathbf{M} \mid \cdot),
$$

where $p(\mathbf{X} \mid \mathbf{M} \cdot)$ is the conditional probability and $p(\mathbf{M} \cdot)$ is the prior probability.

A tip cell moves stochastically towards the source of chemo-attractants and other factors, creating a lumen in the gel (Wood et al., 2011). Its dynamics are modeled with a constant velocity model (Table 1). Stalk cells follow the tip cell along the lumen. Therefore, the propagation of the stalk cells are conditioned on the lumen parameters. We decompose joint state transition model 
in Equation 3 for the stalk cell and lumen as follows

$$
\begin{aligned}
& p\left(\mathbf{x}_{k}^{\mathbf{S}} \mid \mathbf{x}_{k-1}^{\mathbf{S}}, \mathbf{U}_{k}^{\mathbf{X}}, \mathbf{U}_{k}^{\mathbf{M}}\right) \\
& =p\left(\mathbf{X}_{k} \mid \mathbf{X}_{k-1}, \mathbf{M}_{k}, \mathbf{U}_{k}^{\mathbf{X}}, \mathbf{U}_{k}^{\mathbf{M}}\right) p\left(\mathbf{M}_{k} \mid \mathbf{M}_{k-1}, \mathbf{U}_{k}^{\mathbf{X}}, \mathbf{U}_{k}^{\mathbf{M}}\right) \\
& \approx p\left(\mathbf{X}_{k} \mid \mathbf{X}_{k-1}, \mathbf{M}_{k-1}, \mathbf{U}_{k}^{\mathbf{X}}\right) p\left(\mathbf{M}_{k} \mid \mathbf{M}_{k-1}, \mathbf{U}_{k}^{\mathbf{M}}\right) .
\end{aligned}
$$

The first term is associated with the nuclei propagation and is only driven by the previous lumen estimates $\mathbf{M}_{k-1}$, as the current estimate $\left(\mathbf{M}_{k}\right)$ is unavailable. There are no exogenous input modeled, $\left(\mathbf{U}_{k}^{\mathbf{X}}=\emptyset\right)$. The second term is associated with lumen propagation and driven solely by the other input $\mathbf{U}_{k}^{\mathbf{M}}$. This input represents the proteases secreted which degrades the gel matrix to widen the lumen. Hence, we represent the bi-directional cell-lumen interactions with the transition matrix for the stalk cell dynamics equation and with a control input for the lumen dynamics equation.

To constrain the stalk cell estimate within the lumen, its heading is conditioned on the lumen parameters by the desired angle $\alpha_{k-1}$. The angle $\alpha_{k-1}$ is the tangent angle of the lumen wall to the $y$-axis at the location of the cell as shown in Figure 7(b). This angle is a function of both the centerline and radius at two points $a$ and $b$ of the lumen. By denoting

$$
\begin{gathered}
D=y^{a, c}-y^{b, c} \quad E=x^{a, c}+r^{a} \quad F=x^{b, c}+r^{b}, \\
\alpha_{k-1}=\arctan \left(\frac{D_{k-1 \mid k-1}}{E_{k-1 \mid k-1}-F_{k-1 \mid k-1}}\right) .
\end{gathered}
$$

The propagation dynamics of the $i$ th cell position, $x_{k \mid k-1}^{i}$ and $y_{k \mid k-1}^{i}$ at $k$, given the previous state at $k-1$, are dependent on the angle $\alpha_{k-1}$. We model the $z$ coordinate of each $i$ th cell separately with a constant velocity model. The $3 D$ propagation dynamics of its state vector of five dimensions, $\hat{\mathbf{x}}=\left[\hat{x}, \hat{y}, \hat{z}, v^{\hat{x} y}, \hat{v^{z}}\right]^{T}$ is given by:

$$
\hat{\mathbf{x}}_{k \mid k-1}^{i}=\mathbf{F} \hat{\mathbf{x}}_{k-1 \mid k-1}^{i}+\mathbf{w}_{k}^{i}
$$

where

$$
\mathbf{F}=\left[\begin{array}{ccccc}
1 & 0 & 0 & \Delta t \cos \left(\alpha_{k-1}\right) & 0 \\
0 & 1 & 0 & \Delta t \sin \left(\alpha_{k-1}\right) & 0 \\
0 & 0 & 1 & 0 & \Delta t \\
0 & 0 & 0 & 1 & 0 \\
0 & 0 & 0 & 0 & 1
\end{array}\right]
$$

and

$$
\mathbf{w}_{k}=\left[\begin{array}{lllll}
w^{x, i}{ }_{k} & w^{y, i}{ }_{k} & w^{z, i}{ }_{k} & w^{v x y, i}{ }_{k} & w^{v z, i}{ }_{k}
\end{array}\right]^{T},
$$

where $w_{k}^{z^{i}}$ and $w_{k}^{v^{z, i}}$ are white noise. The process noise, $\mathbf{w}_{k}$, is Gaussian with zero mean and covariance $\mathbf{Q}_{k}$.
Note that we correlate the the displacement to velocity with the state transition matrix, F. We propagate the cell nucleus location with the above state transition dynamics, where the angle $\alpha_{k-1}$ is derived from the lumen shape, $\mathbf{M}_{k-1}$. Therefore, the propagation of each nucleus position is dependent on both previous nucleus position and the lumen shape.

We represent the lumen and filopodia by a series of disk center coordinates and radii. Unlike our previous approaches (Ong et al., 2011a,b), we consider the dynamic evolution of lumens based on the computational biology model in (Wood et al., 2012). Endothelial cells secret protease that degrades the gel matrix to widen lumens (Gerhardt, 2006). This can be modeled as a stochastic process in which the lumen radius increases and the lumen centerline drifts. Namely, each lumen disk state vector $\hat{\mathbf{m}}=\left[\hat{x}^{c}, \hat{y}^{c}, \hat{r}\right]^{T}$ is propagated as:

$$
\hat{\mathbf{m}}_{k \mid k-1}^{j}=\hat{\mathbf{m}}_{k-1 \mid k-1}^{j}+\mathbf{u}_{k}^{j}+\mathbf{w}_{k}^{\mathbf{m}, i}
$$

where

$$
\mathbf{u}_{k}^{j}=\left[\begin{array}{lll}
0 & 0 & u_{k}^{r, j}
\end{array}\right]^{T},
$$

and

$$
\mathbf{w}_{k}^{\mathbf{m}, i}=\left[\begin{array}{lll}
w^{x c, j}{ }_{k} & 0 & w^{r, j} k
\end{array}\right]^{T},
$$

where $w_{k}^{x c, j}$ and $w^{r, j}{ }_{k}$ are white noise, and $u_{k}^{r, j}$ is the control input representing the strength of released protease, which degrades the gel matrix over time, increasing the lumen radius. Note that the $y$ coordinate does not change. It is determined by the number of the disks involved $y_{k \mid k-1}^{c, j}=j \Delta y$, where $\Delta y$ is the thickness of each disk. Filopodia extend and contract dynamically when a tip cell burrows into the matrix gel. The filopodia alone are estimated without an evolution model, as no models are currently available in literature.

\subsection{Observation Likelihood}

The propagated PDF, $p\left(\mathbf{x}_{k}^{\mathbf{S}} \mid \mathbf{Z}_{1: k-1}^{3 D}, \mathbf{Z}_{1: k-1}^{2 D}, \mathbf{U}_{1: k-1}^{\mathbf{X}}, \mathbf{U}_{1: k-1}^{\mathbf{M}}\right), \quad$ in Equation 3 can be updated with both nuclei positions and lumen shape observations, to obtain the updated posterior:

$$
\begin{aligned}
& p\left(\mathbf{x}_{k}^{\mathbf{S}} \mid \mathbf{Z}_{1: k}^{3 D}, \mathbf{Z}_{1: k}^{2 D}, \mathbf{U}_{1: k}^{\mathbf{X}}, \mathbf{U}_{1: k}^{\mathbf{M}}\right) \propto \\
& p\left(\mathbf{Z}_{k}^{3 D} \mid \mathbf{x}_{k}^{\mathbf{S}}\right) p\left(\mathbf{Z}_{k}^{2 D} \mid \mathbf{x}_{k}^{\mathbf{S}}\right) p\left(\mathbf{x}_{k}^{\mathbf{S}_{\mid}} \mid \mathbf{Z}_{1: k-1}^{3 D}, \mathbf{Z}_{1: k-1}^{2 D}, \mathbf{U}_{1: k}^{\mathbf{X}}, \mathbf{U}_{1: k}^{\mathbf{M}}\right) .
\end{aligned}
$$

where $p\left(\mathbf{Z}_{k}^{3 D} \mid \mathbf{x}_{k}^{\mathbf{S}}\right)$ and $p\left(\mathbf{Z}_{k}^{2 D} \mid \mathbf{x}_{k}^{\mathbf{S}}\right)$ are the observation likelihood functions for the $3 D$ nuclei and $2 D$ sprout profile observations respectively. The lumen state is not updated with the less-sampled nuclei observations, hence, $p\left(\mathbf{Z}_{k}^{3 D} \mid \mathbf{x}_{k}^{\mathbf{S}}\right)=p\left(\mathbf{Z}_{k}^{3 D} \mid \mathbf{X}_{k}\right)$. The nuclei state update 
is computed with Kalman filtering as the observation likelihood is Gaussian.

To update the joint state with the more-frequently sampled lumen data, we firstly formulate the lumen observation likelihood for the nucleus state. We denote $\xi$ to be the axis orthogonal to the lumen centerline in the $x y$-plane, which passes through the nuclei centroid (shown in 7(b)). In Figure 8, we show that the observation likelihood along the $\xi$ axis varies depending on the lumen width as well as the location of the cell nucleus $\mathbf{x}_{k-1}^{i}$, along the circumference of the lumen. Note that the cell is not located outside of the lumen. We observe that cells tend to move in the middle of narrower lumens. However, in wider lumens, with radii exceeding $10 \mu \mathrm{ms}$, they tend to crawl along one side of the lumen wall. As we only observe the lumen in $2 D$, the observation likelihood is Gaussian when $x$-coordinate of the nuclei is in the center of $\mathbf{m}_{k-1}^{j}$, the cross section of a lumen disk (scenarios (a) and (d)). Otherwise, the distribution is non-Gaussian (scenarios (b) and (c)).

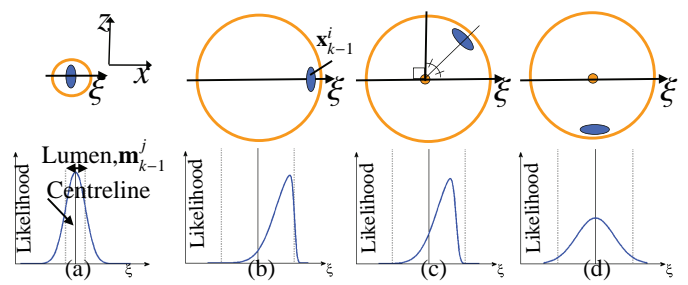

Figure 8: The nuclei likelihood distributions given its current location and lumen radius. (a) shows that a cell, $\mathbf{x}_{k-1}^{i}$, moves in the middle of narrower lumens, whereas for wider lumens ((b),(c) and (d)), the cell is located on the lumen circumference. $\xi$, the axis orthogonal to the lumen centerline in the $x y$-plane, passes through the nuclei centroid. $\mathbf{m}_{k-1}^{j}$ is the cross-section of a lumen disk. If the $x$-coordinate of the nuclei is in the center of the lumen ((a) and (c)), the lumen observation likelihood is Gaussian. Otherwise, the likelihood is skewed by the distance between the nuclei $x$-coordinate and lumen centerline.

It is clear that the general likelihood distribution of each cell $i, p\left(\mathbf{z}_{k}^{2 D, i} \mid \mathbf{x}_{k}^{i}\right)$ is non-Gaussian. Our approach is to model the likelihood as a skewed Gaussian function. This function is a product of a Gaussian function with its cumulative distribution (Liseo and Loperfido, 2004). The skewness is proportional to the cell angular location relative to the $x$-axis, as illustrated in the distributions in Figure 8 . We let $\mathbf{z}_{k}^{2 D, i}$ be the observation of the lumen disks where the $i$-th cell, $\mathbf{x}_{k}^{i}$, resides. Therefore, the likelihood of this observation given the $i$-th cell state is:

$p\left(\mathbf{z}_{k}^{2 D, i} \mid \mathbf{x}_{k}^{i}\right)=2 \mathcal{N}\left(\mathbf{x}_{k}^{i} ; \mathbf{z}_{k}^{2 D, i}, \mathbf{R}_{k}^{2 D, i}\right) \boldsymbol{\Phi}\left(\mathbf{x}_{k}^{i} ; \mathbf{z}_{k}^{2 D, i}, \mathbf{R}_{k}^{2 D, i}, \beta_{k}\right)$, where $\mathcal{N}\left(\mathbf{x}_{k}^{i} ; \mathbf{z}_{k}^{2 D, i}, \mathbf{R}_{k}^{2 D, i}\right)$ is a multivariate Gaussian distribution with mean $\mathbf{z}_{k}^{2 D, i}$ and covariance matrix $\mathbf{R}_{k}^{2 D, i}$ and

$$
\boldsymbol{\Phi}(x ; z, \sigma, \beta)=\frac{1}{\sigma \pi} e^{-\frac{(x-z)^{2}}{2 \sigma^{2}}} \int_{-\infty}^{\beta\left(\frac{x-z}{\sigma}\right)} e^{-\frac{t^{2}}{2}} d t .
$$

The parameters in (15) are:

- $\mathbf{z}_{k}^{2 D, i}=x_{k}^{c, j}+\xi_{k-1}^{i}$ where $\xi_{k-1}^{i}=\left(x_{k-1}^{i}-x_{k}^{c, j}\right) \cdot \cos \theta+$ $\left(y_{k-1}^{i}-y_{k}^{c, j}\right) \cdot \sin \theta$,

- $\mathbf{R}_{k}^{2 D, i}=r_{k}^{j} / 2$,

- $\beta_{k}=r_{k}^{j} \cdot \cos \theta$

where $\beta$ is the skewness factor, $h$ is the lumen disk where the $i$ th cell resides and $\theta$ is the angle between the $\xi$-axis and the $x$-axis as shown in Figure 7(a). We reiterate that the $\xi$-axis is the axis orthogonal to the $j$ th lumen disk centerline. There is no closed form solution to determine the joint maximum-a-posteriori (MAP) when $\beta \neq 0$. Therefore, we need to resort to numerical methods such as particle filters.

A joint cell-lumen state estimate has higher dimensions compared to a cell state estimate alone. Particle filtering degrades quickly when the dimension of the state vector is large (Daum and Huang, 2003). The approach we reported previously (Ong et al., 2011a) does not address this issue. Our solution here is to apply particle filtering for the cell states and Kalman filtering for the lumen states. This technique is known as RaoBlackwell particle filtering (Casella and Robert, 1996) and is used in FastSLAM (Montemerlo and Thrun, 2007). The lumen state is updated in closed form and the cell state, with sampling. This approach is more efficient that using sampling methods for the whole joint state and results in estimates with less variance for the same number of samples.

The combined posterior distribution is represented by a sample set,

$$
\left\{\omega_{k-1}^{(i)}, \mathbf{X}_{k-1}^{(i)}, \mu_{1, k-1}^{(i)}, \Sigma_{1, k-1}^{(i)} \cdots \mu_{l, k-1}^{(i)}, \Sigma_{l, k-1}^{(i)}\right\}
$$

where $i$ is the sample index, $i=1 \ldots, M$ and $l$ is the number of lumen disks. Here, $\omega_{k-1}^{(i)}$ is the sample weight, $\mu_{j, k-1}^{(i)}$ and $\Sigma_{j, k-1}^{(i)}$ are the mean and covariance of the Gaussian distribution associated with the $j$ th disks. Each particle in the set contains the estimated cell state, $\mathbf{X}_{k}^{(i)}$, obtained by first sampling from the marginal cell distribution, $\mathbf{X}_{k-1}$. 
Therefore, the predicted distribution is approximated with a sample set of weighted Gaussians:

$$
\begin{aligned}
& p\left(\mathbf{x}_{k} \mathbf{S}_{\mid} \mathbf{U}_{1: k}, \mathbf{Z}_{1: k-1}\right) \\
& =\int p\left(\mathbf{x}_{k}^{\mathbf{S}} \mid \mathbf{x}_{k-1}^{\mathbf{S}}, \mathbf{U}_{k}\right) p\left(\mathbf{x}_{k-1}^{\mathbf{S}} \mid \mathbf{Z}_{1: k-1}, \mathbf{U}_{1: k-1}\right) d \mathbf{x}_{k-1}^{\mathbf{S}} \\
& \approx \sum_{i=1}^{N} \omega_{k}^{(i)} \mathcal{N}\left(\mathbf{x}_{k}^{\mathbf{S}_{k}} \mid \mu_{k-1}^{(i)}, \Sigma_{k-1}^{(i)}\right),
\end{aligned}
$$

where $\mathbf{x}_{k}^{\mathbf{S}}=\left[\mathbf{X}_{k}, \mathbf{M}_{k}\right], \mathbf{U}_{k}$ is the exogenous inputs driving the migrating cells and lumen and $\mathbf{Z}_{k}$ is the $3 D$ and $2 D$ observation data. The lumen disk radius $r$ and centerline $x^{c}, y^{c}$ are segmented from the transmitted light images. Hence, the observation vector for the $j$ th disk is $\mathbf{z}^{2 D, j}=r^{j}+x^{c, j}$, where $r$ is the radius. Each Gaussian sample $i$ in the Rao-Blackwell particle set is updated with its corresponding lumen disk observation. The sprout may grow at an angle which is not perpendicular to the monolayer. Therefore, we transform the coordinates of the particle set to the frame of the lumen ( $\xi$-axis). The samples are weighted by the likelihood distribution. The result is a weighted Rao-Blackwell sample set representing the posterior distribution. We then convert the distribution back to a Gaussian function by taking the mean and covariance of the particle set. In (Ong et al., 2011b), we compared the performance between a Rao-Blackwell particle update of 100 samples with "naive" particle updates (Gordon et al., 1993). We showed that by using a Rao-Blackwell particle update, the lumen and cell estimates are more accurate compared with using a "naive" particle filter of the same size.

\section{Results on Simulation Data}

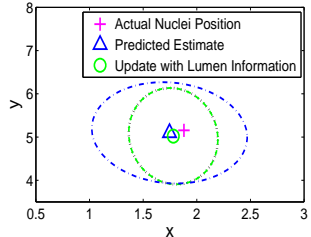

(a) Estimate with lumen update only

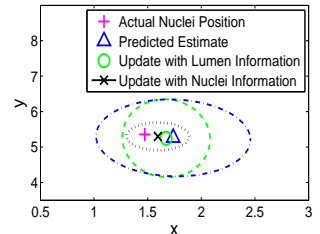

(b) Estimate with lumen and nuclei update
Figure 11: The mean and covariance of the predicted and updated nuclei estimates in the $x y$-plane are shown. (a) shows the accuracy increase of an estimate when updated with only lumen information. (b) shows the increase in accuracy when the predicted estimate is updated with lumen and nuclei information. Results confirm that incorporating lumen information improves the nuclei estimates.

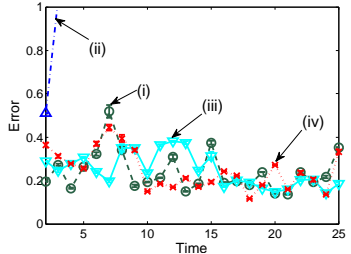

(a) $\kappa=1$.

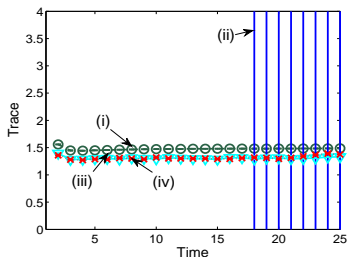

(c) $\kappa=1$.

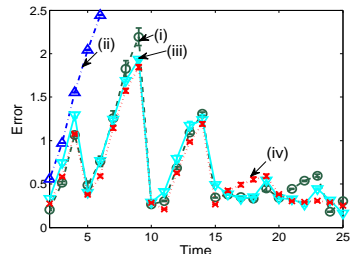

(b) $\kappa=5$.

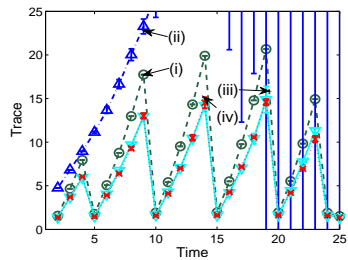

(d) $\kappa=5$.
Figure 12: Our proposed joint nuclei and lumen technique (Case (iv) - red ' $x$ ') results in trajectories with the least Euclidean error ((a) and (b)) to the true nuclei position and filter uncertainty ((c) and (d)). We compared our approach with cell tracking techniques updated with solely nuclei observations (Case (i) - green circles), solely lumen information (Case (ii) - blue $\Delta$ ) and lumen and nuclei observations (Case (iii) - cyan $\nabla$ ). The saw-tooth pattern in (b) and (d) show that error and uncertainty increases when nuclei information is not updated.

We demonstrate the advantage of using lumen information for nuclei localization. Simulations of a cell migrating along a lumen over 25 time-steps were performed. Snapshots are shown in Figure 9. The nuclei centroid was parameterized by its $3 D$ position and velocity. Each disk of the lumen was parameterized by its $2 D$ centreline coordinates and radius. The initial state vector consisted of one nuclei and 9 lumen disks. The initial nuclei parameters were $x=4.1 \mu \mathrm{m}$, $y=0.4 \mu \mathrm{m}, z=5.0 \mu \mathrm{m}, v^{x y}=1.5 \mu \mathrm{m} / \mathrm{hr}$ and $v^{z}=1.5$ $\mu \mathrm{m} / \mathrm{hr}$. The dynamic model given by Equation 8, was used to propagate the mean state vector and covariance matrix. At each time step, the radius and $y$ coordinates of each individual lumen disk were increased randomly. White noise was applied to the $x$ coordinate of each disk. The propagation of each lumen disk estimate, shown in Equation 11, included white noise and a radius control input. Here, for each $j$ th disk, we selected $u_{k}^{r, j}=u_{0}^{r, j} \exp ^{-0.02 k}$ where $u_{0}^{r, j}=0.1 \mu \mathrm{m}$ (Wood et al., 2012). At each time-step, the nuclei $x$ position was adjusted to be at $5 \mu \mathrm{m}$ away from the current lumen wall.

We compared our proposed joint nuclei-lumen technique with three other nuclei only tracking scenarios. The following update computations may be performed:

- (a) nuclei estimate updated with nuclei observations, 


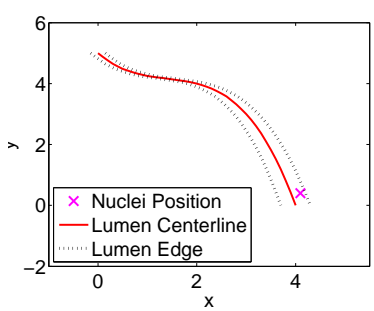

(a) $\mathrm{k}=1$ scenario

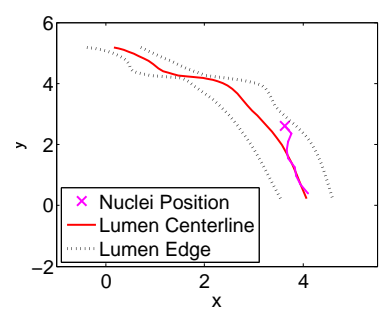

(b) $\mathrm{k}=9$ scenario

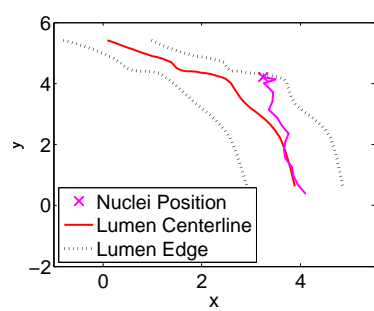

(c) $\mathrm{k}=17$ scenario

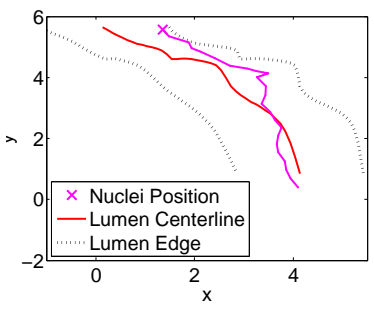

(d) $\mathrm{k}=25$ scenario

Figure 9: Snapshots of simulation at various times, $k$.

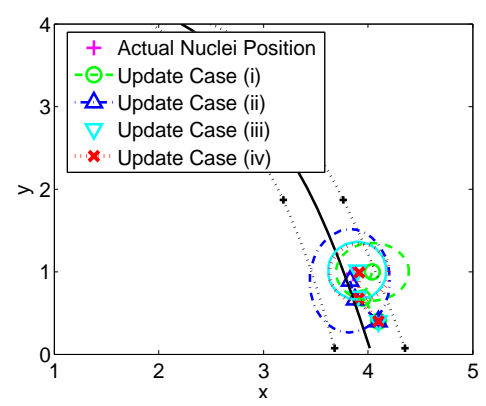

(a) Initial simulation stage.

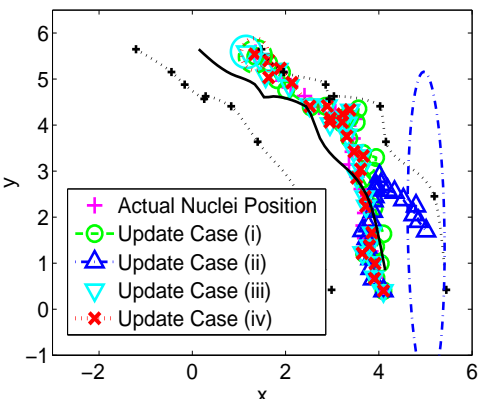

(b) Final results, $\kappa=1$.

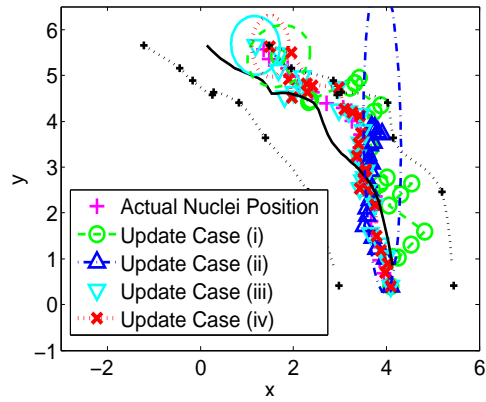

(c) Final results, $\kappa=3$.

Figure 10: Trajectories and final covariance ellipses of the tracking results for four different update scenarios. (a) shows that after three timesteps, the filter solely updated with lumen information (Case (ii) - blue $\triangle$ ), has a largest covariance (blue ellipse) and error, compared to updates that included the $3 D$ nuclei information. (b) shows results when the nuclei and lumen sample rates are the same $(\kappa=1)$. Filtering with both nuclei and lumen updates (Case (iii) - cyan $\nabla$ ) and our proposed joint lumen and nuclei methodology (Case (iv) - red 'x'), provides a slightly better results compared to a filter updated with only $3 D$ nuclei coordinates (Case (i) - green circles). (c) shows that contribution of the lumen information to track accuracy increases when $\kappa=3$.

- (b) nuclei estimate updated with lumen observations,

- (c) lumen estimate updated with lumen observations. This update, if chosen, is performed jointly with (b), using a Rao-Blackwell particle filter update.

In Case (i), the filter is updated with (a). The Case (ii) filter is updated with (b). The nuclei estimate in Case (iii), updated with (a) and (b), shows the contributions of lumen information to filter accuracy. The fourth case jointly tracks the nuclei and lumen, performing all the update computations. By propagating the nuclei and lumen via Equations 8 and 11, this case will show the contribution of biological models to tracking accuracy.

Simulations were reiterated for diverse combinations of sampling rates and observation noise for 20 iterations per case. We let $\kappa$ be the sampling rate ratio,

$$
\kappa=\frac{\text { lumen sampling rate }}{\text { nuclei sampling rate }} \text {. }
$$

As fluorescent images of nuclei are sampled less frequently compared to transmitted light images, $\kappa$ was varied between 1 and 10 . We apply a lumen observation noise of $0.05 \mu \mathrm{m}$ and performed simulations over different levels of nuclei observation noise.

Figure 11 shows the updated mean and covariance estimate, in the $x$ and $y$ plane. Results show that incorporating lumen information improves the nuclei position estimate. Figure 10 shows the tracking results at the initial and final stage of the simulation. At the initial stages, the four different update cases are similar. At the final stages, the filter with solely lumen observation updates fails to track the nuclei. This indicates that nuclei information is required to maintain a good estimate. Figure 10(c) shows the case of reducing nuclei updates to once in three lumen samples. Results indicate that the filter with cell observation updates alone (Case (i)), is less accurate than filters with lumen and nuclei updates (Case (iii)).

We quantified the performance of each filter in terms of the mean and covariance of the estimation error. The 


\begin{tabular}{|c|c|c|c|c|}
\hline & \multicolumn{2}{|c|}{$\begin{array}{c}\text { Update with cell and lumen } \\
\text { observations - Case(iii) }\end{array}$} & \multicolumn{2}{c|}{$\begin{array}{c}\text { Joint cell and lumen } \\
\text { tracking - Case (iv) }\end{array}$} \\
\hline $\begin{array}{c}\text { Noise Level } \\
(\mu \mathrm{m})\end{array}$ & $\begin{array}{c}\text { Mean reduction } \\
\text { in error }\end{array}$ & $\frac{\sum_{k=1}^{I} T r\left[\mathbf{P}_{k \mid k}\right]}{I}$ & $\begin{array}{c}\text { Mean reduction } \\
\text { in error }\end{array}$ & $\frac{\sum_{k=1}^{I} T r\left[\mathbf{P}_{k \mid k}\right]}{I}$ \\
\hline 0.05 & $7.7 \%$ & $21.7 \%$ & $14.3 \%$ & $22.8 \%$ \\
0.10 & $8.2 \%$ & $22.8 \%$ & $13.1 \%$ & $22.9 \%$ \\
0.25 & $6.8 \%$ & $21.6 \%$ & $14.1 \%$ & $23.1 \%$ \\
0.50 & $14.3 \%$ & $23.0 \%$ & $21.8 \%$ & $21.2 \%$ \\
1.00 & $17.0 \%$ & $20.7 \%$ & $22.8 \%$ & $17.7 \%$ \\
\hline
\end{tabular}

Table 2: We show the contribution of lumen information and our proposed motion models (Case (iii) and (iv)) as the nuclei noise increases. We compare the mean Euclidean error and mean trace of these filters to filters with only nuclei observations (Case (i)). Larger reductions in error are shown, when the nuclei noise level increases, whereas the reduction in covariance trace is the similar.

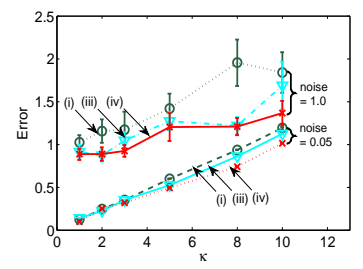

(a) Average Error

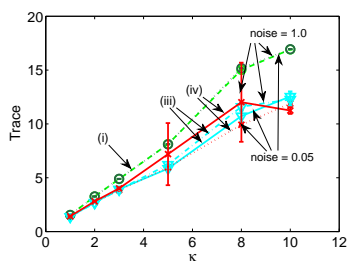

(b) Average Trace
Figure 13: We validate that our proposed joint nuclei and lumen estimates (Case (iv) - red 'x') results in (a) a lower average over different lumen to nuclei sampling rates $\kappa$, for two different nuclei observation noise, $0.05 \mu \mathrm{m}$ and $1.0 \mu \mathrm{m}$, and (b) a lower corresponding information average mean trace (sum of diagonals of the covariance). Our technique was compared to nuclei estimation techniques updated with nuclei information only (Case (i) - green circles) and with nuclei and lumen information (Case (iii) - cyan $\nabla$ ).

simulations were performed at lumen-to-nuclei sample rates of $1 \leq \kappa \leq 10$. The cell centroid and lumen observation noise was $0.05 \mu \mathrm{m}$. The mean and covariance of the Euclidean error and trace of the error covariance matrix were obtained based on 20 iterations of the simulation. Figure 12 shows the error and trace over time for $\kappa=1$ an $\kappa=5$. We show that using the joint nuclei and lumen state for tracking provides the best results. Results also show that the error and trace are larger when $\kappa$ is larger. The error and trace increases when no nuclei observations are made as shown when $\kappa=5$. However, we show that updates with lumen information reduce this uncertainty increase.

Figure 13 show that lumen information contributes more significantly when the lumen to nuclei observation sampling rate decreases. We also examined the effect of the nuclei observation noise by increasing the nuclei observation noise from 0.1 to 1.0 . In these scenarios, the lumen observation noise was held constant at 0.05 . As the nuclei noise increases, the estimates are less ac-

\begin{tabular}{|l|c|c|c|c|c|}
\hline & \multicolumn{5}{|c|}{ Dataset } \\
\hline Number of ... & 1 & 2 & 3 & 4 & 5 \\
\hline initial cells & 2 & 3 & 5 & 4 & 3 \\
proliferations & 0 & 0 & 0 & 0 & 0 \\
cell deaths & 0 & 0 & 0 & 0 & 0 \\
cells migrated into gel & 0 & 0 & 5 & 2 & 7 \\
time points acquired & 27 & 27 & 15 & 20 & 17 \\
\hline
\end{tabular}

Table 3: Details of experimental data used.

curate and have larger uncertainty. The variance in the results also increases as the nuclei observation noise increase.

Table 2 quantifies the contribution of lumen information with respect to noise. The error and trace of the filter updated with only nuclei information (Case (i)) is subtracted from the two filters that have lumen information contribution (Cases (iii) and (iv)). We also show that contribution of lumen information increases when the nuclei noise level increases. By jointly tracking lumens in addition to nuclei (Case (iv)) and predicting the estimates via dynamic equations from biological models, in Equations 8 and 11, we obtain more accurate lumen estimates.

\section{Results on Experimental Data}

We now demonstrate using experimental data, the advantages of combining lumen and filopodia information for nuclei localization. Our methodology was applied to five different data sets of cells migrating in the gel. We ran time-lapse experiments at 30 minute intervals over a period of 8 to 15 hours, according to our experimental protocol. Cell viability is compromised when longer time-lapses are performed. The least complex dataset, Dataset 1, shown in Figures 14(a) and 14(b), consists of two cells migrating in the gel. This data set does not 


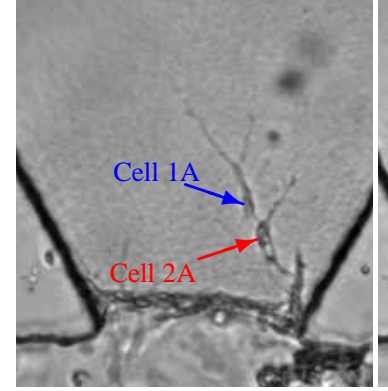

(a) Start of cell migration

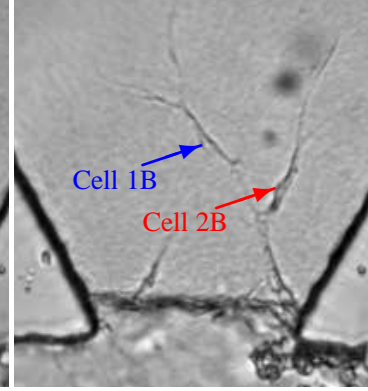

(b) End of cell migration

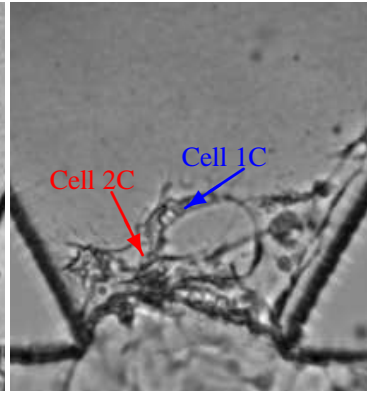

(c) Start of branching

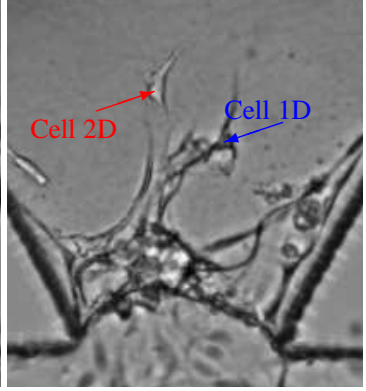

(d) End of branching

Figure 14: $2 D$ transmitted light images of sprouting from time lapse image acquisition.

contain any lumens. A more complex data set, Dataset 3, is shown in Figures 14(c) and 14(d). At the start of image acquisition, there was a lumen and tip cell (Cell 1C). During the timelapse, a stalk cell (Cell 2C) following this tip cell changed its phenotype. It became another tip cell (Cell 2D) and a branch in the sprout was formed. Table 3 provides a summary of the five datasets used.

Observations of the nuclei in $3 D$ were updated at every 90 minutes. $2 D$ observations of the sprout profile were determined at every 30 minutes. Our methodology, (Case (iv)), was compared with standard filters that only track the nuclei state with updates from nuclei observations only (Case (i)), sprout profile observations (Case (ii)), and sprout profile and nuclei observations (Case (iii)). We applied white noise of zero mean and covariance of 0.5 pixels for 20 iterations to the nuclei centroid and sprout observations. At the start, each filter was initialized based on two consecutive nuclei observations (at 30 intervals).

The stained nuclei fragments of dead cells are smaller in size compared to healthy cells (Chae et al., 2007). In our image segmentation module (Appendix A.1), smaller clustered pixels which are under 100 pixels are removed. Dead cell nuclei are not segmented as observations. As a result, the track is not updated and therefore is removed. To track proliferation, we use multiple hypothesis tracking. We create separate hypothesis to track multiple cell candidates that fall into a validation gate. To identify that proliferation has occurred, the Mahalanobis distance measure is applied to cluster the hypothesis. If the distance measure between clusters exceeds the chi-square threshold, the filter would be duplicated and used to track the two daughter cells.

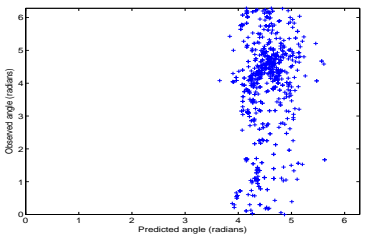

(a) Predicted and actual orientation

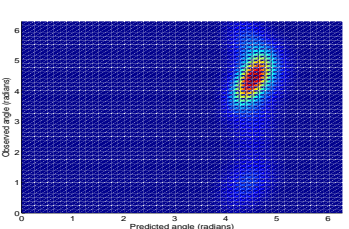

(b) Distribution function of (a)
Figure 15: In (a) we compare our predicted cell orientation and actual cell orientation for approximately 300 cell updates. Each point is one cell update. In (b) the distribution of the points in (a) show that the accuracy of our prediction reflects a Gaussian function.

\subsection{Validation of Predicted Cell Models and Update using Sprout Profile}

We compare our methodology with a Kalman filtering solution updated with $3 D$ nuclei samples, available at a frequency of 30 minutes. Frequent sampling of stained nuclei affects cell viability, and therefore, we would prefer to acquire these $3 D$ fluorescence images less frequently. Our proposed algorithms help us to achieve this goal. Although we take $3 D$ samples only every 90 minutes, we can still obtain cell trajectories at relatively the same accuracy, by integrating $2 \mathrm{D} \mathrm{lu}-$ men information recorded every 30 minutes. In other words, combining $2 D$ lumen information successfully compensates for the fewer $3 D$ nuclei samples. We compared the predicted nuclei orientation $\left(\alpha_{k-1}\right)$ described in Equation 7, with the nuclei migration orientation obtained from Kalman filtering solution with more samples, for all our five datasets in Figure 15. We show that our nuclei prediction model compares well to the actual position for approximately 300 cell updates. We also showed in (Ong et al., 2011b), with experimental data of sprout information, that the Rao-Blackwellized parti- 


\begin{tabular}{|l|c|c|}
\hline & $\begin{array}{c}\text { Nuclei candidates } \\
\text { with no sprout profile }\end{array}$ & $\begin{array}{c}\text { Nuclei candidates } \\
\text { at filopodia }\end{array}$ \\
\hline Dataset 1 & 8 & 0 \\
Dataset 2 & 46 & 13 \\
Dataset 3 & 95 & 3 \\
Dataset 4 & 59 & 5 \\
Dataset 5 & 247 & 2 \\
\hline
\end{tabular}

Table 5: False nuclei candidates detected with sprout information

cle update resulted in a better covariance approximation compared to an extended Kalman filtering update.

\subsection{Comparison of tracking results}

Figures 16 and 17 show the nuclei trajectories at the end-point. Results show that jointly tracking both nuclei and sprout profile provide higher accuracy compared to updates of the nuclei state alone. Figure 18 show the results of the trace results, $\operatorname{Tr}\left[\mathbf{P}_{k \mid k}^{x^{i}}\right]$, for each nuclei state, $\mathbf{x}_{k \mid k}^{i}$, in the Dataset 1 (from Figure 14(b)). The saw-tooth pattern indicates that covariance uncertainty increases when nuclei updates are not made. However, our methodology has the smallest increase in filter uncertainty. Our tracking technique results in a lower total innovation, as shown in Figure 18(c). The total innovation is average measurement residual, $\sum_{i=1}^{N}\left(\mathbf{z}_{k}^{3 D, i}-\right.$ $\left.\mathbf{H} \hat{\mathbf{x}}_{k: k-1}^{i d x(i)}\right) / N$, where $N$ is the number of times that a $3 D$ measurement is updated to a track, and $i d x(i)$ is the associated track for observation $i$. The lower the innovation, the better the association of predicted estimate to the observation. Table 4 shows the nuclei estimate mean and covariance and number of incorrect track associations for our three tracking approaches. We show that combining $2 D$ lumen information successfully compensates for the fewer $3 D$ nuclei samples by comparing to the Kalman filtering solution with more $3 D$ nuclei samples, explained in Section 4.1. We determine that errors below the average nucleus diameter of $10 \mu \mathrm{m}$ (14.4 pixels) are acceptable (Jean et al., 2004).

\subsection{Further advantages of incorporating sprout infor- mation}

In addition to tracking accuracy, incorporating lumen information improves identification of nuclei candidates and detection of cell phenotype. Sprout profiles are also used to identify false positives of nuclei. Nuclei candidates are segmented from noisy fluorescent images. There may be false positives among these candidates, which include nuclei that are outside the sprout profile

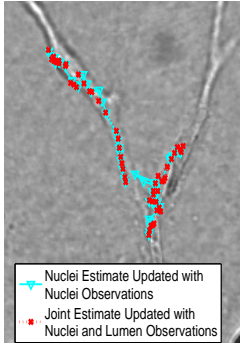

(a)

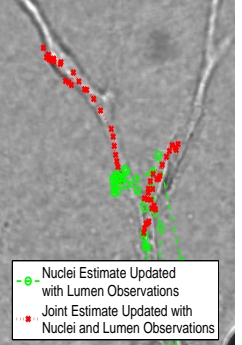

(b)

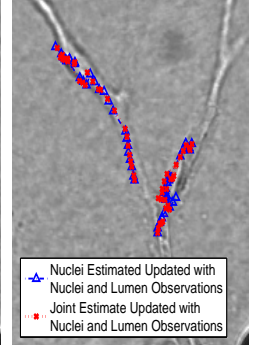

(c)
Figure 16: Comparison tracked results for Dataset 1 in Figures 14(a) and 14(b). We show that a joint nuclei and sprout Rao-Blackwell particle filtering (Case (iv)) outperforms with separate filtering updated with (a) nuclei information only (Case (i)), (b) lumen information only (Case (ii)) and (c) nuclei and lumen information (Case (iii)). The nuclei to sprout profile sampling rate is $1: 3$.
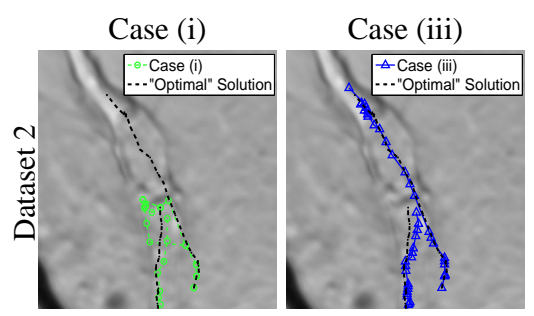

Case (iv)
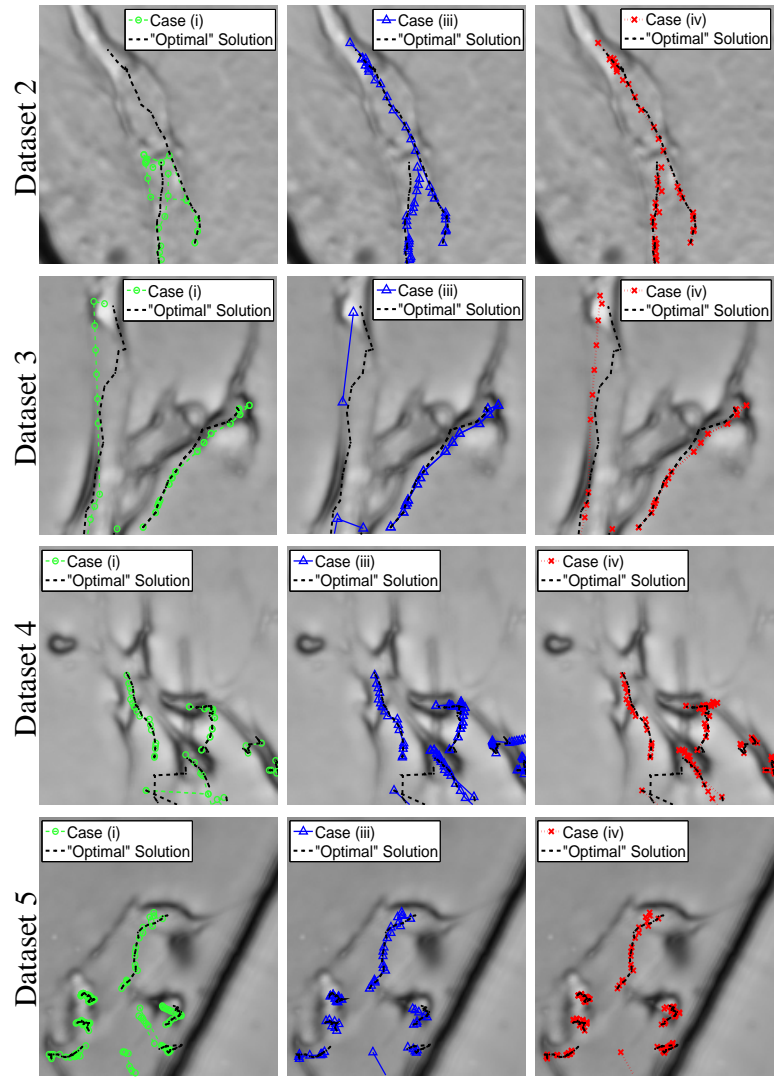

Figure 17: Comparison of nuclei trajectories to the optimal solutions without lumen information (black dotted line). These are Kalman smoothing solutions with nuclei observations updated at 30-minute intervals. The nuclei to sprout profile sampling rate is $1: 3$. The left column shows nuclei tracking with nuclei information only. The middle column shows nuclei tracking with nuclei and lumen information. The right column shows the joint nuclei and sprout tracking. We show the stalk cells tracked with our methodology have closer solutions to the optimal one, as well as less incorrect associations. 


\begin{tabular}{|l|c|c|c|c|}
\hline & Dataset & $\begin{array}{c}\text { Mean error } \\
\text { (in pixels) }\end{array}$ & $\begin{array}{c}\text { Error covariance } \\
\text { (in pixels) }\end{array}$ & $\begin{array}{c}\text { Incorrect track } \\
\text { associations }\end{array}$ \\
\hline Joint cell and & 1 & 5.34 & 23.97 & 0 \\
sprout tracking & 2 & 3.69 & 10.35 & 0 \\
Case (iv) & 3 & 8.21 & 86.94 & 3 \\
& 4 & 1.98 & 6.35 & 0 \\
\hline & 5 & 1.64 & 4.46 & 0 \\
Tracking with cell and & 1 & 5.86 & 22.58 & 0 \\
sprout observations & 2 & 4.35 & 13.43 & 0 \\
Case (iii) & 3 & 11.84 & 173.24 & 9 \\
& 4 & 3.73 & 23.57 & 0 \\
\hline & 5 & 1.84 & 3.00 & 0 \\
Tracking with only & 1 & 6.44 & 49.23 & 0 \\
cell observations & 2 & 7.17 & 254.3 & 0 \\
Case (i) & 3 & 11.84 & 173.24 & 3 \\
& 4 & 3.73 & 23.57 & 1 \\
\hline
\end{tabular}

Table 4: Comparison of the Euclidean error between the three tracking systems in reference to the Kalman filtering solution with more $3 D$ nuclei samples. We also show the number of incorrect track associations. Errors below the nucleus diameter of $10 \mu \mathrm{m}$ (14.4 pixels) are reasonable. Our joint nuclei and sprout tracking solution results in more trajectories with errors below this amount.

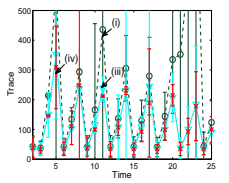

(a) Cell 1 Trace

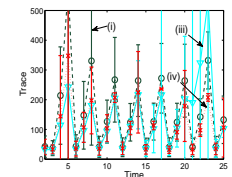

(b) Cell 2 Trace

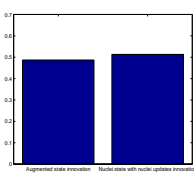

(c) Total Innovation
Figure 18: Comparison of the covariance trace for the cell tracked shown in (a) and (b). (c) shows that sum of the innovation for our method is better for Dataset 1 .

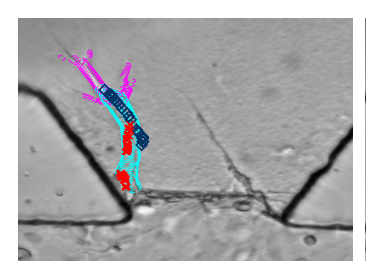

(a) Data Set 2 Final Solution

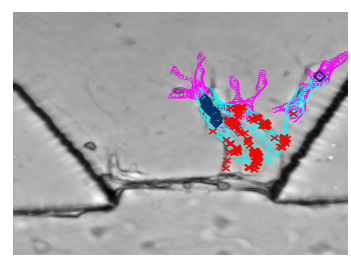

(c) Data Set 4 Final Solution

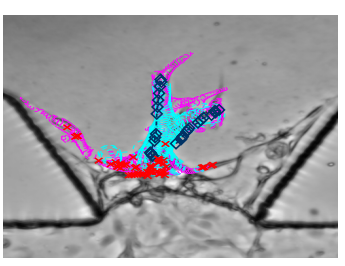

(b) Data Set 3 Final Solution

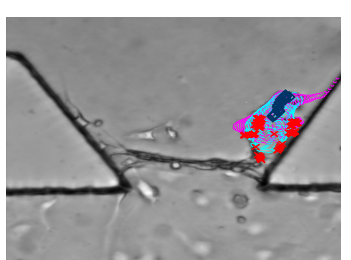

(d) Data Set 5 Final Solution
Figure 19: The final lumen, filopodia and cell trajectories for Dataset 2 to 5. By tracking lumens (cyan disks) and filopodia (magenta disks), we can classify cells to tip cells (green diamonds) and stalk cells (red crosses). or attached to a filopodia. These false positives are rejected. In Table 5, we show the tracking error and number of false positives detected from five datasets applied.

Figure 19 shows the final lumen, filopodia and cell trajectories for four of our datasets. By identifying and tracking cell phenotype, we can automatically classify tip cells (dark green diamonds) and stalk cells (red crosses). Figure 20 shows that we can obtain obtain lumen growth details and change of a stalk cell to a tip cell from Dataset 3 using our technique. Other cell tracking methods (Debeir et al., 2005; Padfield et al., 2009; Bise et al., 2011) do not track gel deformation. Hence, those technique would fail to detect branching and tip/stalk cell phenotype change compared to our methods. Furthermore, cells in the $3 D$ microenvironment interact with the surrounding gel. Therefore, it is important to observe both cell movements and gel remodeling at the same time, not only angiogenesis studies but also in applications of myogenesis and stem cell differentiation.

\subsection{Visualization of results}

To visualize the sprout in $3 D$, we assume that the $\mathrm{z}$ axis centerline coordinates is the average of the $\mathrm{z}$-plane of the cell coordinates. We also assume a circular or constant radius at each centerline point. The representative of the visualization for Dataset 2, is shown in Figure 21. The segmented tip and stalk cells are represented by the orange and purple spheres using a visualization software (IMARIS, Bitplane). 


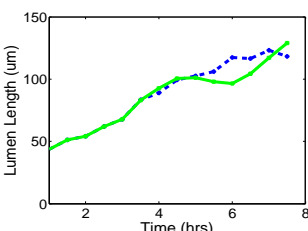

(a) Lumen Elongation

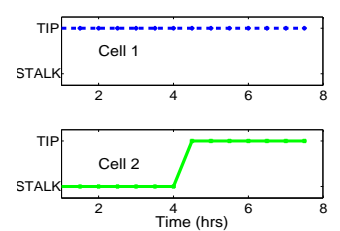

(b) Cell Phenotype Change
Figure 20: Lumen elongation and cell phenotype change in Dataset 3. In (a), the blue dotted line shows the elongation of the lumen created by Cell 1C in Figure 14(c). When stalk cell (Cell 2C) becomes a tip cell, branching occurs and the elongation of the new lumen branch is shown by the green solid line. (b) shows the phenotype of Cell $1 \mathrm{C}$ (blue dotted line) and Cell 2C (green solid line) over time.

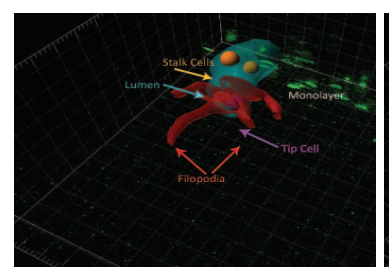

(a) Visualization at image start

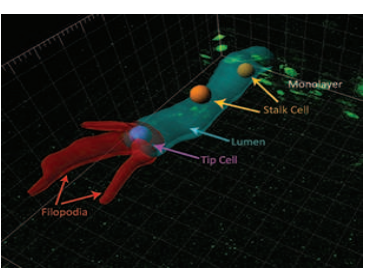

(b) Visualization at image end
Figure 21: Visualization of the joint track sprout profile and cells for Dataset 2. Tip cells are identified in purple and the stalk cells in orange.

\section{Conclusion}

In this paper, we proposed a new approach to jointly estimate cell and sprout locations by combining $3 \mathrm{D}$ fluorescent images of stained nuclei with $2 D$ information from transmitted light images of gel deformation. To our knowledge, we are the first to develop an algorithm to incorporate sprout information to locate cells more accurately. During $3 D$ migration, cells remodel and deform the surrounding gel. Specifically, in angiogenesis, tip cells create lumens in the gel. Stalk cells tend to follow the tip cell, crawling along the lumen wall. Biological models describing these interactions are incorporated into our framework. We demonstrate through analysis of synthetic and experimental data that our approach indeed allows us to localize cells more precisely. Although we have developed our methodology specifically for angiogenic sprouting, our framework can be generalized and applied to other fields which involve interactions between cells and gel matrix.

Our tracking techniques may fail if the sprout to nuclei sampling rate is too large. Nuclei observations are required for successful tracking. Poor nuclei and filopodia observations may also be another cause of failure.
Nuclei may be poorly stained and images may be acquired when the microscope is misaligned or the sample is poorly illuminated. Furthermore, we may fail to segment nuclei that are close together. In future, we will also segment and track cell shape statistics. Cell shape information can be used to detect boundaries of overlapping nuclei.

\section{Acknowledgements}

The authors would like to thank Levi B. Wood for assistance in performing cell culturing and timelapse imaging. This research was supported by the National Research Foundation Singapore through the Singapore MIT Alliance for Research and Technology's BioSystems and Micromechanics Inter-Disciplinary Research programme.

\section{Appendix A. Image Segmentation Workflow}

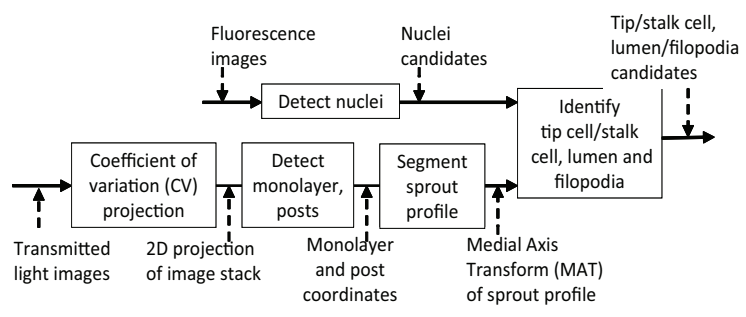

Figure A.22: The modules for image segmentation of fluorescent and transmitted light images. We acquire the centroid of each nuclei candidate in the "Detect nuclei" module. We enhance the regions of high pixel intensity variations by a coefficient of variation (CV) projection. These includes cell, monolayer and microfluidic post regions. We segment the monolayer and post coordinates from the CV projected image. The sprout region and cells in the gel are identified from the monolayer and post data. We then derive the Medial Axis transformations (MAT) of the sprout region and obtain circular disks representing the sprout shape. Finally, we classify nuclei to tip and stalk cells and sprout profile to lumen and filopodia using rule-based techniques.

Confocal images of migration regions were acquired at approximately 30 minute intervals from two different illumination sources. These are $3 D$ fluorescent images of stained cells and $2 D$ transmitted light images of the extracellular matrix. We used a 20x objective lens magnification and a 0.75 numerical aperture to acquire these images. These images are obtained on the same microscope at the same time, and hence are registered by default. The 12-bit image size obtained is 512 by 512 , acquired with a $2 \mu$ s laser dwell time per pixel. The focus of this work is on studying cell sprouting. Therefore, 
our interest lies in tracking cell trajectories and sprout above the monolayer. Our software was developed using MATLAB (2010a, The MathWorks, Natick, MA). Figure A.22 shows an overview of our image segmentation workfolk, which comprises of (a) nuclei segmentation, (b) sprout profile segmentation, (c) monolayer detection, (d) medial axis transform (MAT) approximation and (e) tip/stalk cell, lumen and filopodia identification.
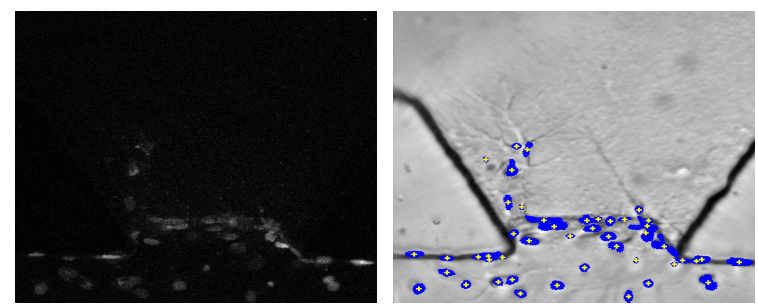

(a) Maximum intensity projec- (b) Segmented nuclei centroid tion of acquired fluorescence im- from image (a) age

Figure A.23: (b) shows the segmented nuclei (blue), and nuclei centroid (yellow '+') from (a) against its corresponding transmitted light image in the $x y$ plane.

\section{Appendix A.1. Nuclei Candidate Segmentation}

Nuclei candidates are segmented from the fluorescence images. We convolve each image with a Gaussian kernel, followed by a Laplacian operator to improve nuclei region contrast. K-means clustering (Seber, 1984) was then applied to classify the image pixels into either cell or background regions. These cell pixels are clustered based on connected components clustering (Qidwai and Chen, 2010). Clusters that are typically nuclei fragments of dead cells, non-specific binding or noise are smaller in size (less than 100 pixels), and hence removed. To separate larger clusters containing more than one nuclei, we apply level sets (Chan and Vese, 2001) on the clusters. The nuclei candidates are parameterized by the $3 D$ centroid of each cluster. Figure A.23(b) shows the segmentation results from Figure A.23(a).

\section{Appendix A.2. Lumen Candidate Segmentation}

In bright field images, the cells that comprise the sprout and monolayer are nearly transparent. Despite the out-of-focus blur in these images, pixel intensities inside the sprout vary more than the background as the microscopy focus changes. Hence prior to segmentation, we enhance the image contrast by increasing the intensity differences between cell and background areas. Our approach is to construct a $2 D$ projection of the
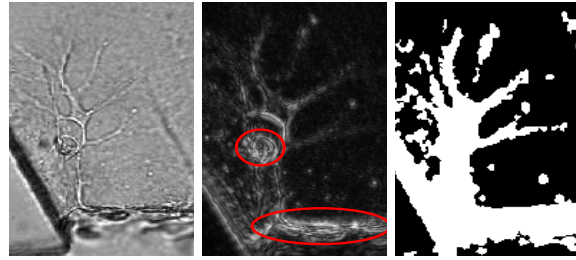

(a) Original image tion of image (a) tion

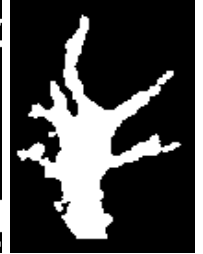

(d) Thresholded Image
Figure A.24: The coefficient of variation (CV) projection (b) of (a) improves the contrast between the sprout and background. We show that the monolayer and cell regions (red circles) are more clearly contrasted than the background. In (c), the vessel network is clearly defined after Otsu thresholding from (b). (d) shows the sprout region after smaller clusters, the monolayer and device posts are removed.

image stack by calculating the coefficient of variation (CV) of each image stack (Selinummi et al., 2009). An example of this projection is shown in Figure A.24(b). Intensity thresholding is then performed on the projection, to obtain the results in Figure A.24(c). We cluster the thresholded pixels in Figure A.24(c) and remove smaller clusters, as these clusters represent the nonuniform texture of the collagen gel. We then identify the monolayer and post in Appendix A.3 to exclude the device posts from the sprout profile. Figure A.24(d) shows the sprout region extracted above the monolayer from the image in Figure A.24(c).

\section{Appendix A.3. Segmentation of Matrix Region}

Features segmented from the transmitted light image, such as the trapezoidal device posts and monolayer are used to determine the gel region. We apply Sobel edge detection (Canny, 1986) followed by Hough transform (Qidwai and Chen, 2010) to detect lines representing the posts in these images. In our experimental images, the slopes of each post angle are either between the angles of 25 to 35 degrees or -25 to -35 degrees. Hence, the longest lines within the two angular thresholds were selected as post boundary lines (as shown in Figure A.25(a)). From these lines, we numerically search for the separating boundary line between the pixel intensity distribution of the CV projected image.

We optimize our approach to monolayer detection using similar methods to posts detection. We linearly combine the nuclei and transmitted light intensity signals in the image. Next, we determine the coefficients of 4-point cubic spline function to describe the monolayer. Our algorithm iterates different combinations of these coefficients, to find the pixel coordinates from the 

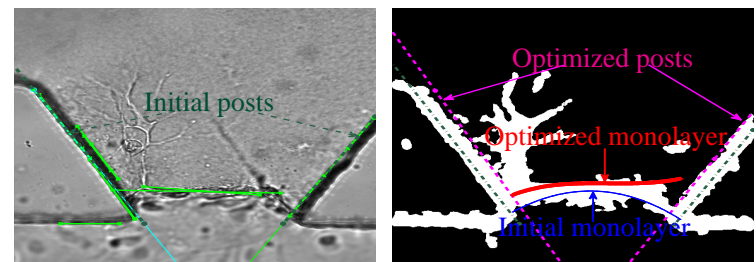

(a) Initial posts and monolayer (b) Optimized posts and monodetected

Figure A.25: (a) device posts (green) found using Hough transform. In (b), the monolayer function (blue line) is obtain from maximizing the fluorescence signals between the posts. We perform a search for the optimal monolayer boundary (red line) by maximizing the boundary between the pixel intensity distribution of the $\mathrm{CV}$ projected image. We also perform a search for the line function (dotted line) that maximizes the pixel intensity distribution between the posts and gel region.

spline function that returns the highest image intensity. We then numerically search for the optimal spline parameters that maximize the boundary between the pixel intensity distribution of the $\mathrm{CV}$ projected image. Results of the optimizer search is shown in Figure A.25(b).

\section{Appendix A.4. Shape representation using a medial axis transform network}

Tracking a binary sprout profile grid, shown in Figure A.24(d), is highly inefficient. Instead, we use a more compact representation that preserves the sprout shape; a Medial Axis Transform (MAT), which represents the image with a centreline and radius. The radius is the distance between the centerline and the boundary of the image. Therefore, we are able to reconstruct the original shape for visualization (Sherbrooke et al., 1996).

We convert the sprout profile image to a MAT via Delaunay triangulation. Delaunay triangles are computed from the sprout boundary points. The medial axis transform center and radii for each triangle is the associated circumcircle. Figure A.26(a) shows the medial axis points and their corresponding Delaunay triangles. Certain points in the medial axis may be classified into junction points and end points. The Delaunay triangle of a corresponding junction point (labeled in blue) have three adjacent triangles. The corresponding triangle of an end point (shown in pink) has one connected triangle. The remaining of triangles have two adjacent triangles and form parts of a seam. In Figure A.26(a), the green lines are seams between junction points and the red lines are seams between a junction point and end points or two end points.
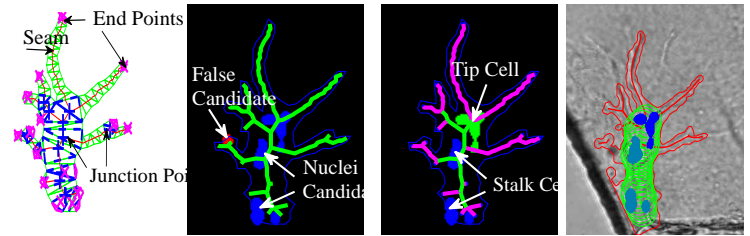

(a) Classifica- (b) Detection of (c) Tip and (d) Lumen and tion of Medial false nuclei can- stalk cell filopodia identiAxis Points didates identification fication

Figure A.26: (a) The junction, end points, seams and their corresponding Delaunay triangle. (b) Segmented nuclei candidates are associated to seams. Nuclei candidates associated to seams with smaller radii and unassociated nuclei (shown by the red patches) are rejected. (c) Tip cells are identified by the number of seams with small radii linked, and its location on the seam links. (d) The segmented profile and centreline of the filopodia are shown in red and the extracted lumen is shown in green. Lumens are identified by seam links with radii exceeding a threshold and by the existence of a tip cell.

\section{Appendix A.5. Identification of tip and stalk cell and lu- men and filopodia}

To identify tip/stalk cell phenotype, lumens and filopodia, we combine information from nuclei candidates with the medial axis transforms. Each nucleus is associated with medial axis seam links. As a result, cell candidates located on filopodia (labeled in red in Figure A.26(b)) are excluded. One condition to identify tip cell is filopodia activity. Another condition is that a tip cell would furthest from the monolayer along its seam link than the other associated stalk cells as shown in Figure A.26(c). We classify the seam (or parts of a seam) to lumens and filopodia based on the its radius and location of an associated tip cell. shown in Figure A.26(d).

\section{References}

Abercrombie, M., Heaysman, J.E., Pegrum, S.M., 1970. The locomotion of fibroblasts in culture: III. Movements of particles on the dorsal surface of the leading lamella. Experimental Cell Research 62, 389-398.

Agin, G., Binford, T., 1976. Computer description of curved objects. IEEE Transactions on Computers C-25, 439-449.

Bise, R., Yin, Z., Kanade, T., 2011. Reliable cell tracking by global data association, in: IEEE International Symposium on Biomedical Imaging, pp. $1004-1010$.

Borau, C., Kamm, R., Garca-Aznar, J., 2011. Mechano-sensing and cell migration: a 3D model approach. Physical Biology 8, 066008.

Bunyak, F., Palaniappan, K., Nath, S.K., Baskin, T.I., Dong, G., 2006. Quantitative cell motility for in vitro wound healing using level set-based active contour tracking, in: In: Proceedings of the Third IEEE International Symposium Biomedical Imaging (ISBI), pp. 1040-1043.

Canny, J., 1986. A computational approach to edge detection. IEEE Transactions on Pattern Analysis and Machine Intelligence PAMI8, 679-698. 
Casella, G., Robert, C.P., 1996. Rao-Blackwellisation of sampling schemes. Biometrika 83, 81-94.

Chae, Y., Kim, S.J., Kim, D., 2007. Screening of apoptotic genes by morphological analysis of high throughput fluorescence microscopy images, in: Proceedings of the 2007 Frontiers in the Convergence of Bioscience and Information Technologies, IEEE Computer Society, Washington, DC, USA. pp. 16-20.

Chan, T.F., Vese, L.A., 2001. Active contours without edges. IEEE Transactions on Image Processing 10, 266-277.

Cukierman, E., Pankov, R., Stevens, D.R., Yamada, K.M., 2001. Taking cell-matrix adhesions to the third dimension. Science 294, 1708-1712.

Daum, F.E., Huang, J., 2003. Curse of dimensionality and particle filters. Proceedings of IEEE Aerospace Conference 4, 4-1979-41993.

Debeir, O., Van Ham, P., Kiss, R., Decaestecker, C., 2005. Tracking of migrating cells under phase-contrast video microscopy with combined mean-shift processes. IEEE Transactions on Medical Imaging 24, 697-711.

Farahat, W., Wood, L., Zervantonakis, I., Schor, A., Ong, S., Neal, D., Kamm, R., Asada, H., 2012. Ensemble analysis of angiogenic growth in three-dimensional microfluidic cell cultures. PloS ONE 7, e37333.

Florin, C., Paragios, N., Williams, J., 2006. Globally optimal active contours, sequential monte-carlo and on-line learning for vessel segmentation, in: In: Proceedings of European Conference Computer Vision, pp. 476-489.

Folkman, J., Haudenschild, C., 1980. Angiogenesis in vitro. Nature $288,551-556$.

Gerhardt, H., 2006. VEGF and Endothelial Guidance in Angiogenic Sprouting. VEGF in Development. chapter 6. pp. 68-78.

Godinez, W., Lampe, M., Wörz, S., Müller, B., Eils, R., Rohr, K., 2009. Deterministic and probabilistic approaches for tracking virus particles in time-lapse fluorescence microscopy image sequences. Medical Image Analysis 13, 325-342.

Gordon, N.J., Salmond, D., Smith, A., 1993. Novel approach to nonlinear/non-Gaussian Bayesian state estimation. Radar and Signal Processing, IEE Proceedings F, 140(2), 107-113.

Jean, R., Gray, D., Spector, A., Chen, C., 2004. Characterization of the nuclear deformation caused by changes in endothelial cell shape. J Biomech Eng 126, 552-558.

Kim, M., Kim, C., Wood, L., Neal, D., Kamm, R., Asada, H., 2012. Integrating focal adhesion dynamics, cytoskeleton remodeling, and actin motor activity for predicting cell migration on 3D curved surfaces of extracellular matrix. Integrative Biology 4, 1386-1397.

Kirbas, C., Quek, F.K.H., 2000. A review of vessel extraction techniques and algorithms. ACM Computing Surveys 36, 81-121.

Leonard, J., Durrant-Whyte, H., 1991. Simultaneous map building and localisation for an autonomous robot, in: IEEE/RSJ Int. Workshop on Intelligent Robots and Systems (IROS '91), Osaka, Japan. pp. 1442-1447.

Lesage, D., Angelinib, E.D., Bloch, I., Funka-Leaa, G., 2009. A review of 3D vessel lumen segmentation techniques: Models, features and extraction schemes. Medical Image Analysis 13, 819845 .

Li, K., Miller, E., Chen, M., Kanade, T., Weiss, L., P.G., C., 2008. Cell population tracking and lineage construction with spatiotemporal context. Medical Image Analysis 12, 546-566.

Liseo, B., Loperfido, N., 2004. Default Bayesian analysis of the skewnormal distribution. Journal of Statistical Planning and Inference $136,373-389$

Montemerlo, M., Thrun, S., 2007. FastSLAM: A Scalable Method for the Simultaneous Localization and Mapping Problem in Robotics. Springer-Verlag Berlin and Heidelberg GmbH \& Co. K.

Montrucchio, B., Lamberti, F., Gamba, A., Serini, G., 2005. Track- ing endothelial cells during blood vessel networks assembly using active contours, in: IEEE Workshop on Signal Processing Systems Design and Implementation.

Ong, L.L., Ang, M.H., Asada, H.H., 2011a. Simultaneous tracking of cell nuclei and conduit parameters from time-lapse confocal microscopy images, in: Proceedings of the 8th IEEE International Symposium on Biomedical Imaging: From Nano to Macro (ISBI 2011), pp. 1011-1014.

Ong, L.L.S., Wood, L.B., Ang, M.H., Asada, H.H., 2011b. Stochastic tracking of migrating live cells interacting with 3D gel environment using augmented-space particle filters, in: Proceedings of the IEEE/RSJ International Conference on Intelligent Robots and Systems (IROS), San Francisco, CA, USA. pp. 3931-3936.

Padfield, D., Rittscher, J., Thomas, N., Roysam, B., 2009. Spatiotemporal cell cycle phase analysis using level sets and fast marching methods. Medical Image Analysis 13, 143-155.

Pellot, C., Herment, A., Sigelle, M., 1994. A 3D reconstruction of vascular structures from two X-ray angiograms using an adapted simulated annealing algorithm. IEEE Transactions on Medical Imaging 13, 48-60.

Petrie, R.J., Yamada, K.M., 2012. At the leading edge of threedimensional cell migration. Journal of cell science 125, 59175926.

Qidwai, U., Chen, C.H., 2010. Digital Image Processing: An Algorithmic Approach with MATLAB. Chapman \& Hall/CRC, 2010.

Sacan, A., Ferhatosmanoglu, H., Coskun, H., 2008. Celltrack: An open-source software for cell tracking and motility analysis. Bioinformatics 24, 1647-1649.

Sakar, M.S., Neal, D., Boudou, T., Borochin, M.A., Li, Y., Weiss, R., Kamm, R.D., Chen, C.S., Asada, H.H., 2012. Formation and optogenetic control of $3 \mathrm{~d}$ engineered skeletal muscle bioactuators. Lab on a Chip 12, 4976-4985.

Seber, G.A.F., 1984. Multivariate Observations. John Wiley \& Sons, Inc.

Selinummi, J., Ruusuvuori, P., Podolsky, I., Ozinsky, A., Gold, E., Yli-Harja, O., Aderem, A., Shmulevich, I., 2009. Bright field microscopy as an alternative to whole cell fluorescence in automated analysis of macrophage images. PLoS ONE 4, e7497.

Sherbrooke, E., Patrikalakis, N., Brisson, E., 1996. An algorithm for the medial axis transform of 3D polyhedral solids. IEEE Transactions on Visualization and Computer Graphics 2, 44-61.

Smal, I., Draegestein, K., Galjart, N., Niessen, W., Meijering, E., 2008. Particle filtering for multiple object tracking in dynamic fluorescence microscopy images: Application to microtubule growth analysis. IEEE Transactions on Medical Imaging 27, 789-804.

Truc, P.T., Khan, M.A., Lee, Y.K., Lee, S., Kim, T.S., 2009. Vessel enhancement filter using directional filter bank. Computer Vision and Image Understanding 113, 101-112.

Vickerman, V., Blundo, J., Chung, S., Kamm, R., 2008. Design, fabrication and implementation of a novel multi-parameter control microfluidic platform for three-dimensional cell culture and real-time imaging. Lab on a Chip, The Royal Society of Chemistry 8, 14681477.

Wood, L., Ge, R., Kamm, R., Asada, H., 2012. Vessel elongation rate is inversely related to diameter in in vitro angiogenesis. Integrative Biology 4, 1081-1089.

Wood, L., Kamm, R., Asada, H., 2011. Stochastic modeling and identification of emergent behaviors of an endothelial cell population in angiogenic pattern formation. The International Journal of Robotics Research 30, 659-677.

Wörz, S., Rohr, K., 2007. Segmentation and quantification of human vessels using a 3-d cylindrical intensity model. In Proceedings IEEE Transactions Image Processing 16, 1994-2004.

Yamada, K., Cukierman, E., 2007. Modeling tissue morphogenesis and cancer in 3d. Cell 130, 601-610. 
Yang, X., Li, H., Zhou, X., 2006. Nuclei segmentation using markercontrolled watershed, tracking using mean-shift, and kalman filter in time-lapse microscopy. IEEE Transactions on Circuits and Systems I: Regular Papers 53, 2405-2414.

Zana, F., Klein, J.C., 2001. Segmentation of vessel-like patterns using mathematical morphology and curvature evaluation. IEEE Transactions on Image Processing 10, 1010-1019.

\section{Vitae}

Dr. Lee-Ling (Sharon) Ong is a postdoctoral associate at the Singapore-MIT Alliance for Research and Technology (SMART) Centre. She received her doctoral degree in Mechatronics Engineering from the University of Sydney, Australia in 2008. Her work focuses on integrating biological models with experimental data from time-lapse microscopy. Her research interests include image processing, stochastic modeling and multiagent systems.

Dr. Justin Dauwels is an Assistant Professor with School of Electrical and Electronic Engineering at Nanyang Technological University (NTU), Singapore. He obtained a $\mathrm{PhD}$ degree in electrical engineering at the Swiss Polytechnical Institute of Technology (ETH) in Zurich in 2005. Prior to joining NTU, he was a research scientist during 2008-2010 in the Stochastic Systems Group (SSG) at the Massachusetts Institute of Technology (MIT). His research interests are in Bayesian statistics, iterative signal processing, and computational neuroscience. He enjoys working on realworld problems, often in collaboration with medical practitioners.

Dr. Marcelo H. Ang, Jr. is an Associate Professor of Mechanical Engineering at the National University of Singapore. He received the M.Sc. and Ph.D. degrees in Electrical Engineering from the University of Rochester, Rochester, NY, in 1986 and 1988, respectively. His research interests span the areas of robotics, mechatronics, and applications of intelligent systems methodologies. He teaches both at the graduate and undergraduate levels in the following areas: robotics; creativity and innovation, applied electronics and instrumentation; advanced computing; product design and realization, and special topics in mechatronics. He is also active in consulting work in these areas.

Dr. H. Harry Asada is Ford Professor of Engineering with the Department of Mechanical Engineering at Massachusetts Institute of Technology (MIT), USA. He earned his Ph.D. in Precision Engineering from Kyoto University in 1979 and started his faculty position at MIT in 1982. His expertise range from robotics, Biomedical Engineering, dynamic systems and control, information technology to design and manufacturing. $\mathrm{He}$ is also the Director, d'Arbeloff Laboratory for Information Systems and Technology and current Head of Control, Instrumentation, and Robotics in Department of Mechanical Engineering at MIT. 